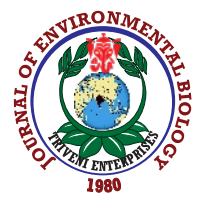

\title{
Temporal variations in abundance and species richness of phytoplankton with emphasis on diatoms in the subtidal waters of Umm Al-Namil Island, north-western Arabian Gulf of the ROPME Sea Area
}

\author{
Matrah Al-Mutairi ${ }^{1}$, M.N.V. Subrahmanyam ${ }^{1,2}$, Mohammad Ali $^{1 *}$, Sasini Isath ${ }^{1}$, Mohammad A. AlAwadi ${ }^{1}$, P. Niranjan Kumar ${ }^{1}$, \\ Khaled Al-Hebini ${ }^{1}$ and Samira A. S. Omar ${ }^{1}$ \\ ${ }^{1}$ Environment and Life Sciences Research Center, Kuwait Institute for Scientific Research, Safat-13109, Kuwait \\ ${ }^{2}$ Head Quarters, Regional Organization for the Protection of the Marine Environment (ROPME), Safat-13124, Kuwait \\ *Corresponding Author Email : mohammad.awad.ali@gmail.com
}

Paper received: 19.04 .2019

Revised received: 28.09 .2019

Accepted: 31.01 .2020

Abstract

Aim: The present study aimed to understand the physico-chemical factors affecting the abundance and species richness of phytoplankton communities, with emphasis on diatoms at the end of the intertidal zone and start of subtidal zone.

Methodology: Water quality and phytoplankton community species richness and abundance were measured in the subtidal waters of Umm Al-Namil Island, Kuwait Bay. Kruskal-Wallis statistical approach was used to measure the effect of various environmental parameters on abundance and species richness as well as abundance-species richness relationships using $\mathrm{R}$ statistical software.

Results: The physico-chemical parameters showed variations at each sampling event as well as within the same season. Total phytoplankton mean abundance values in summer $\left(22.26 \pm 0.66\right.$ ind. $\left.5 l^{-1}\right)$ and winter $\left(21.63 \pm 0.52\right.$ ind. $\left.5 l^{-1}\right)$ did not significantly vary and the lowest mean abundance was observed during spring $\left(26.36 \pm 0.33\right.$ ind $\left.\left..5\right|^{-1}\right)$. Species abundance showed clear pattern with the events, resulting in somehow a negative relationship. Mean species richness was highest during autumn $(24.59 \pm 1.51)$, while no significant difference was observed between spring (11.09 \pm 0.33$)$, winter $(10.47 \pm 0.51)$ and summer (10.11 \pm 0.66$)$. The results indicate that phytoplankton species richness and species abundance in Umm Al-Namil Island varied temporally in response to fluctuations in environmental conditions.

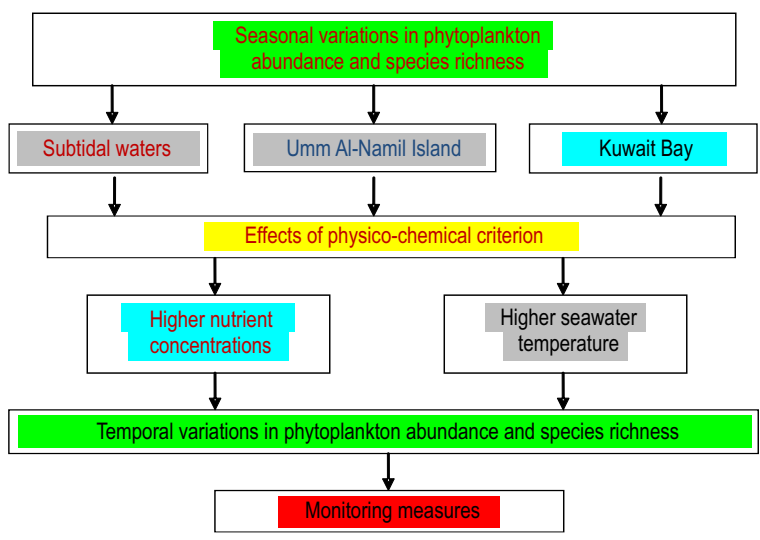

Interpretation: The end of intertidal zone and beginning of subtidal zone is affluent in diverse forms of phytoplankton species, specifically diatoms since Kuwait Bay is a eutrophic area mainly due to sewage and urbanization. Therefore, a temporal monitoring is required in order to observe any changes in either physico-chemical or biological factors with time and that will help in future conservation and restoration programs. Conservation approaches should be considered seriously since some diatoms like Melosira moniliformis, Gyrosigma diminutum, Fragilaria martyi, Hemidiscus cuneiformis, Planktoniella sol and Amphiprora plicata have been observed only at one event despite abundance and season.

Key words: Kuwait Bay, Phytoplankton, Species richness, Subtidal waters, Umm Al-Namil Island

How to cite : Al-Mutairi, Matrah, M.N.V. Subrahmanyam,Mohammad Ali, Sasini Isath, Mohammad A. AlAwadi, P. Niranjan Kumar, Khaled AlHebini and Samira A. S. Omar: Temporal variations in abundance and species richness of phytoplankton with emphasis on diatoms in the subtidal waters of Umm Al-Namil Island, north-western Arabian Gulf of the ROPME Sea Area. J. Environ. Biol., 41, 1470-1485 (2020). 


\section{Introduction}

Phytoplankton plays a vital role in maintaining the ecological evenness of the coastal ecosystems for sustaining all other forms of life by producing oxygen and food (Khan, 2003). Understanding the abundance, the community composition of phytoplankton can contribute further perceptive of structure and functioning of pelagic ecosystem and benefit in administration of fisheries. Biodiversity is an imperative prerequisite for the preservation of ecosystem maintenance providing diversified assistance to human prosperity (Hoekstra et al., 2012). With relevance to phytoplankton biodiversity, these assistances may reach from encouraging primary productivity and supplies food (via the trophic cascade) to coordinate water quality and climate (e.g., via nutrient and carbon fixation, oxygen production), in addition to creative and health form (e.g., preventing cyanobacteria influence and blooms in water bodies used for recreational purposes). Besides, biodiversity curtails adverse feedback to environmental changes by strengthening the resistance and flexibility of ecosystems (McNaughton, 1977; Naeem and Li 1997; Chapin et al., 2000).

Nonetheless, biodiversity is drop off at a speedy dimension (Hooper et al. 2005; Worm et al., 2006) and this decrease is further increased by man-made climate change and related increasing temperatures on a comprehensive proportion (Lovejoy and Hannah 2006; IPCC 2007). The perception of phytoplankton distribution with implication to spatial pattern is important to persuade the status of the ecosystem structure and functioning. The spatial mapping of phytoplankton assists to resolve hotspots established on abundance and diversity (Badsi et al., 2012; Nassar and Hgarib, 2014; Ahmed and Wanganeo, 2015). Measures of diversity are periodically seen as indicators of the stature of ecological systems. Phytoplankton diversity has relevance with yield in ecology (Newall et al., 2011; Vallina et al., 2014). In extent, the diversity index may be used for habitat characterization (de Domitrovic and de Neiff, 2007; Polikarpov et al, 2008; Cardoso et al., 2012). Phytoplankton patterning varies across ecosystems, have large year-to-year alternation, and display annual cycles of biomass fluctuation and habituation strength (Scheffer 1991; Winder and Cloern 2010; Al-Yamani et al., 2017; Devlin et al., 2019).

Temperature is an important parameter controlling phytoplankton production rates, nutrient utilization, and spatial and temporal distribution in marine systems. In extension to aspects such as grazing pressure or nutrient availability, phytoplankton taxa react adversely to various temperature systems (Canale and Vogel, 1974; Butterwick et al., 2005). With overall average surface temperatures envisaged to increase by 1.1-6.4 ${ }^{\circ} \mathrm{C}$ within the next 100 years (IPCC 2007), water temperatures will benefit this tendency, leading to alteration in the thermal system of many seawater habitats (Webb and Nobilis 2007) and strengthen transition in both phytoplankton community arrangement and distribution in markedly sensitive ecosystems like modest water bodies or tiny islands.
This study was performed during low tide at the start of the subtidal zone (the area at the end of the intertidal zone where subtidal waters start) to $10 \mathrm{~cm}$ depth off Umm Al -Namil Island, as it forms an important transition area between intertidal and subtidal zones. The aim of the study herein was to understand the physico-chemical factors affecting the abundance and species richness of phytoplankton at the end of the intertidal zone and the start of subtidal zone off UmmAl-Namil Island.

\section{Materials and Methods}

Site description: Umm Al-Namil Island (29²2'43.4496"N $\left.47^{\circ} 52^{\prime} 4.3176 " \mathrm{E}\right)$ is one of the small islands among the nine islands present within the territorial waters of Kuwait in the northwestern part of the Arabian (= Persian) Gulf of the ROPME (Regional Organization for the Protection of the Marine Environment) Sea Area. ROPME Sea Area is divided into three sub-areas: inner, middle and outer sea areas. The study area herein lies within the inner ROPME Sea Area. The island is around $600 \mathrm{~m}$ away from Kuwait mainland. It is situated at the southwestern corner of Kuwait Bay along the northwestern side of Sulaibikhat Bay (Fig. 1). It is oriented in a northeast-southwest direction and has a drumstick shape (Al-Zamel et al., 2007).

The island narrows to $75 \mathrm{~m}$ in the southwestern side and is approximately $800 \mathrm{~m}$ long and $300 \mathrm{~m}$ wide at the eastern side. Along the eastern side of the main tidal channel of the island, the maximum water depth is about 4-5 $\mathrm{m}$ (Al-Zamel et al., 2007). The island is bounded by well-developed tidal flats. Supratidal flats consist of gypsum and anhydrite, which form most of the coastal dunes in the area and the intertidal flat is hard and accompanied by oyster mounds, whereas the subtidal flat is soft muddy sand with high productivity (Al-Zamel et al., 2007).

Sampling and analysis: Phytoplankton samples were collected during the period 2016-2017 at monthly intervals covering 4 seasons representing autumn (October and November), winter (December, January, and February), spring (March and April), and summer (May, June, July, August and September). Sampling was performed during low tide at the start of the subtidal zone (the area at the end of the intertidal zone where subtidal waters start) at depth up to $10 \mathrm{~cm}$ off Umm Al-Namil Island. A unique number was assigned for each sampling (Table 1).

Samples were collected in one-liter sampling bottle and poured into a 20-microns mesh for five times. Samples preserved in glutaraldehyde-formaldehyde solution (1:1 vol) were allowed to settle for $48 \mathrm{hrs}$ in $1.5 \mathrm{l}$ measuring cylinders. The settling and siphoning procedure (Utermöhl, 1958) with settling chambers were used for microscopic analysis of plankton samples. Organisms collected from $25 \mathrm{ml}$ out of $100 \mathrm{ml}$ of the concentrated bottom samples were further settled on $25 \mathrm{ml}$ Utermöhl chambers for another $24 \mathrm{hrs}$ before carriying out the taxonomic analysis. A $25 \mathrm{ml}$ sub-sample was poured into a settling chamber, and left to 
settle for $24 \mathrm{hrs}$. The base plate was then removed from the chamber and a slide placed over the top of the base plate into which the planktonic organisms had settled. The samples were enumerated using a Leica inverted microscope (model number DMi1). The counting and identification of phytoplankton was carried out based on the method reported by Tomas (1997).

The abundance of each plankton was calculated as individuals presente in $5 \mathrm{I}$ (ind. $5 \mathrm{I}^{-1}$ ), since 5 liters of water were collected and filtered through $20 \mu$ mesh as explained above to catch the phytoplankton for this study. All individuals in the samples were counted and identified. Water temperature, $\mathrm{pH}$, salinity, dissolved oxygen were measured in the subtidal sampling location using amultiparameter field meter (Orion Star A326, Thermo-Fisher Scientific STARA3260 series). Water samples were collected and brought back to the laboratory for nutrient analysis, i.e., nitrate $\left(\mathrm{NO}_{3}\right)$, nitrite $\left(\mathrm{NO}_{2}\right)$, phosphate $\left(\mathrm{PO}_{4}\right)$ and Silicates $\left(\mathrm{SiO}_{4}\right)$. Standard analytical procedures (MOOPAM, 2010) were used for measuring the concentrations of the aforementioned nutrients using the UV-Vis
Spectrophotometer (Beckman coulter A23615 Du 720 GeneralPurpose Spectrophotometer).

Statistical analyses: A non-parametric Kruskal-Wallis statistical approach was used to test the effect of various environmental parameters on abundance and species richness as well as abundance-species richness relationships using $\mathrm{R}$ statistical software version 3.2.3 (R Core Team, 2016).

\section{Results and Discussion}

The physico-chemical parameters varied within the same sampling season at each sampling event (Fig. 2 and 3 ). Dissolved oxygen (DO) concentration ranged between $5 \mathrm{mg} \mathrm{l}^{-1}$ in winter (at event 5 and 6 ) to $8.2 \mathrm{mgl}^{-1}$ (at events 4 in winter and 12 in summer) (Fig. 2A). The highest mean concentration of DO was observed during autumn $\left(7.59 \pm 0.029 \mathrm{mg} \mathrm{l}^{-1}\right)$, while the lowest was observed during winter $\left(5.75 \pm 0.25 \mathrm{mgl}^{-1}\right)$ (Fig. $3 \mathrm{C}$ ). However, the mean DO value in winter did not significantly differ from that of summer $\left(6.49 \pm 0.11 \mathrm{mgl}^{-1}\right)$ and spring $\left(6.16 \pm 0.087 \mathrm{mgl}^{-1}\right)$ (Fig.

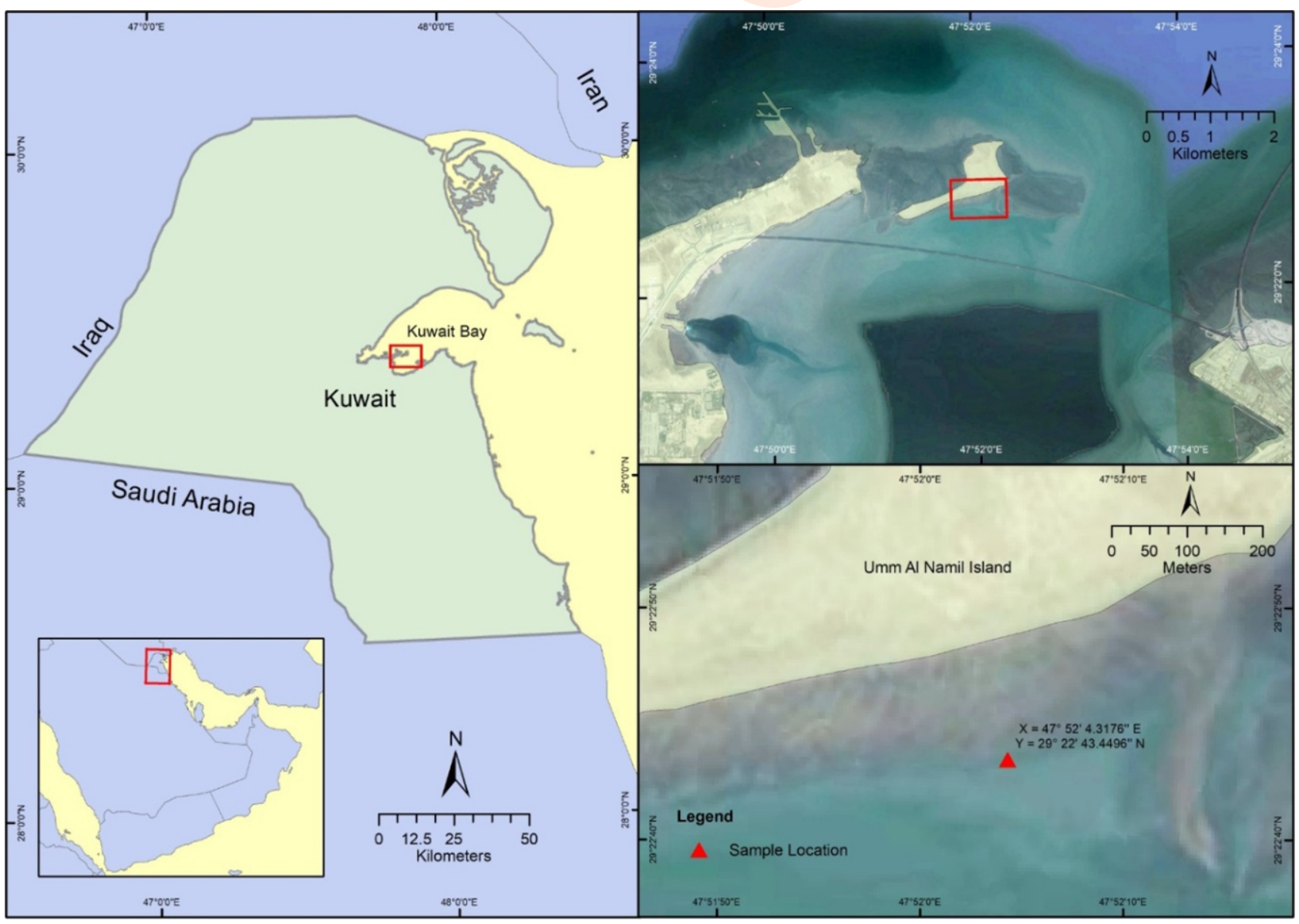

Fig. 1: Map of Kuwait showing the location of Umm Al-Namil Island in Kuwait Bay, North-western Arabian (= Persian) Gulf, along with the sampling location represented by a red triangle at the start of the subtital zone $\left(29^{\circ} 22^{\prime} 43.44966^{\prime \prime} \mathrm{N} 47^{\circ} 52^{\prime} 4.3176\right.$ "E) during low tide. 
Table 1: Sampling months during the study period in the subtidal area of Umm Al-Namil Island. The "Event" column refers to sampling event. Each event has been assigned a unique number that refers to the month and year where sampling occurred. This was done in order to make figure representation tidier. Table also shows seasons related to each month

\begin{tabular}{lll}
\hline Month, Year & Season & Event \\
\hline September, 2016 & Summer & 1 \\
October,2016 & Autumn & 2 \\
November, 2016 & Autumn & 3 \\
December, 2016 & Winter & 4 \\
January, 2017 & Winter & 5 \\
February,2017 & Winter & 6 \\
March, 2017 & Spring & 7 \\
April, 2017 & Spring & 8 \\
May, 2017 & Summer & 9 \\
June, 2017 & Summer & 10 \\
July, 2017 & Summer & 11 \\
August,2017 & Summer & 12 \\
\hline
\end{tabular}

3C). Sea surface temperature (SST) ranged between 7.2 to $29.4^{\circ} \mathrm{C}$ at events 4 and 11 in winter and summer, respectively (Fig. 2B). A consistent pattern of variations in SST was visible within the season (Fig. 2B, 3A). The mean SST was $29.10 \pm 0.11$, $21.36 \pm 0.029,17.82 \pm 0.087,9.51 \pm 0.25^{\circ} \mathrm{C}$ in summer, autumn, spring, and winter, respectively (Fig. $3 A$ ).

Salinity ranged between 42 to 47.1 psu at events 3 and 12 during autumn and summer, respectively (Fig. 2C). Salinity did not significantly vary among spring (45.96 $\pm 0.15 \mathrm{psu})$, summer ( $45.79 \pm 1.67 \mathrm{psu})$, and winter (44.29 $\pm 0.98 \mathrm{psu})$, but was lowest during autumn (42.16 $\pm 0.15 \mathrm{psu}$ ) (Fig. 3B). The $\mathrm{pH}$ values ranged between 5.2 to 10 at events 4 and 8 during winter and spring seasons (Fig. 2D). The pH level was highest during spring (9.73 \pm $0.054)$ compared to other seasons, which did not significantly vary $6.74 \pm 0.072,6.15 \pm 0.054$, and $5.66 \pm 0.047$ during summer, winter, and autumn, respectively) (Fig. 2D, 3D). Nitrate level ranged from 0.02 (events 6 in winter and 7 in spring) to $0.8 \mathrm{mg} \mathrm{l}^{-1}$ at event 4 in winter (Fig. 2E). The level of nitrate was almost same throughout the events 2, 6-12 (Fig. 2E) without significant variations unlike events 1, 3, 4-5 (Fig. 2E). Nitrate levels were highest during winters $0.38 \pm 0.58 \mathrm{mg} \mathrm{l}^{-1}$ at and lowest in spring being near zero, with no significant difference between autumn $\left(0.16 \pm 0.019 \mathrm{mg} \mathrm{l}^{-1}\right)$ and summer $\left(0.17 \pm 0.016 \mathrm{mg} \mathrm{l}^{-1}\right)$ (Fig. 2E). Phosphates showed almost a consistent pattern with events but the lowest value was observed in summer at events 9 and 10 being $0.19 \mathrm{mg} \mathrm{l}^{-1}$ and highest value was observed at event 6 in winter being $0.7 \mathrm{mgl}^{-1}$ (Fig. 2F). Generally, the level of phosphates varied with season being, $0.465 \pm 0.0371 \mathrm{mg} \mathrm{l}^{-1}$ (winter), $0.20 \pm$ $0.011 \mathrm{mgl}^{-1}$ (autumn), $0.23 \pm 0.011 \mathrm{mgl}^{-1}$ (summer), and $0.196 \pm$ $0.0011 \mathrm{mgl}^{-1}$ (spring) (Fig. 2F, 3H) but was highest in winter being $0.5 \pm 0.058 \mathrm{mgl}^{-1}$ (Fig. 2F, 3H).

Silicates did not show a consistent pattern with events but it was highest at event 12 in summer $\left(2.71 \mathrm{mg} \mathrm{l}^{-1}\right)$ and lowest at event 6 in winter $\left(0.61 \mathrm{mg} \mathrm{l}^{-1}\right)$ (Fig. $\left.2 \mathrm{G}\right)$. Highest mean level of silicates was observed during autumn $\left(0.95 \pm 0.016 \mathrm{mg} \mathrm{l}^{-1}\right)$ and lowest during winter $\left(1.032 \pm 0.079 \mathrm{mg} \mathrm{l}^{-1}\right)$, however, no significant variation between summer $\left(2.17 \pm 0.077 \mathrm{mg} \mathrm{l}^{-1}\right)$ and spring $\left(1.55 \pm 0.020 \mathrm{mg} \mathrm{l}^{-1}\right)$ (Figs. 2G, 3E). Nitrites were lowest in autumn at events 2 and 3 with the same value $\left(0.02 \mathrm{mgl}^{-1}\right)$, while the highest value $\left(0.58 \mathrm{mg} \mathrm{l}^{-1}\right)$ was recorded at event 4 in winter (Fig. $2 \mathrm{H})$. There was no significant difference in mean nitrites level between summer $\left(0.077 \pm 0.004 \mathrm{mgl}^{-1}\right)$ and spring $(0.073 \pm$ $0.0023 \mathrm{mg} \mathrm{l}^{-1}$ ) (Fig. 2H, 3G). Phytoplankton abundance (ind. $5 \mathrm{I}^{-1}$ ) was highest at event 2 in autumn (50 ind. $\left.5 \mathrm{l}^{-1}\right)$ and lowest at event 12 in summer ( 13 ind. $\left.5 l^{-1}\right)$ (Fig. 2l). Mean abundance was highest during autumn (48.59 \pm 0.22 ind. $\left.\left.5\right|^{-1}\right)$, followed by spring (26.36 \pm 0.33 ind $\left.5 \mathrm{l}^{-1}\right)$. Mean phytoplankton abundance during summer $\left(22.26 \pm 0.66\right.$ ind. $\left.5 l^{-1}\right)$ and winter $\left(21.63 \pm 0.52\right.$ ind. $\left.5 l^{-1}\right)$ did not significantly vary, and the lowest mean abundance was observed in the latter season (Fig. 2l). Species richness was highest at event 2 (autumn) being 50, while the lowest species richness was observed in summer at event 12 being 3 (Table 5, Fig. $2 \mathrm{~J}$ ). Mean species richness was highest during autumn (24.59 \pm 1.51$)$, while no significant difference was observed between spring (11.09 \pm 0.33$)$, winter (10.47 \pm 0.51$)$, and summer (10.11 \pm 0.66$)$ (Fig. 3J). Like abundance, the values of species richness at events 5 to 10 did not significantly vary, and both richness and abundance were lowest at event 12 in summer (Fig. 2I,J). Both species richness and abundance had significantly varied with season (Fig. 2I, J).

The effect of season as well as the effect of physicochemical parameters was significant on both abundance and species richness of different phytoplankton groups (Tables 3-5). The effect of abundance on species richness was significant, and the maximum percentage of diatom observed was $92 \%$ and the lowest was $38.46 \%$ (Fig 5). Previous studies on the Arabian Gulf showed that it is exposed to potential impacts by recent urban developments (Ibrahim and El Samra, 1987; Al-Muzaini and Jacob, 1996; Subba-Rao et al., 1999; Nour El-Din and Al-Khayat, 2005; QMEMP, 2005; Sheppard et al., 2010; Al-Yamani et al., 2017; Devlin et al., 2019), also, nitrogen limitation plays a role in the functioning of this ecosystem (Quigg et al., 2013).

The Arabian Gulf ecosystem is currently facing loss of precise habitat to development, pollution due to discharge of toxic chemicals by industry, with particularly the ones relevant to oil exploration, invasive species from ballast waters, and eutrophication (e.g., Ibrahim and El Samra, 1987; Al-Muzaini and Jacob, 1996; Subba-Rao et al., 1999; Nour El-Din and AlKhayat, 2005; Qatar Marine Environment Monitoring, 2005; Sheppard et al., 2010). Over-and-above, "brine" discharged from desalination plants with salinities > 75 psu will escalate salinity in most hypersaline water bodies (Abdul Azis et al., 2003; Lattemann and Hopner, 2008; Sheppard et al., 2010). So, increased salinity could lead to higher phytoplankton diversity but lower biomass and lower production (Polikarpov et al., 2008). Succession of phytoplankton species composition was observed and it was due to the changes in the physico-chemical 
Table 2: Kruskal-Wallis test showing the effect of season, seawater temperature, salinity, dissolved oxygen concentration, nitrates, nitrites, phosphates, and silicates on phytoplankton abundance. The effect of season on abundance was significant (Chi-Square $=109.16, \mathrm{df}=3, p=2.2 \times 10^{-16}$ ), as well as the effect of temperature on the abundance (Chi-Square $\left.=145, \mathrm{df}=11, p=2.2 \times 10^{-16}\right)$. The effect of salinity on abundance was also significant (Chi-Square $=145, \mathrm{df}=11, p=2.2 \times 10^{-16}$ ), as well as the effect of dissolved oxygen on abundance (Chi-Square $\left.=138.19, \mathrm{df}=9, p=2.2 \times 10^{-16}\right)$. The effect of $\mathrm{pH}$ was also significant (Chi-Square $=134.6, \mathrm{df}=9, p=2.2 \times 10^{-16}$ ) along with those of nitrates (Chi-Square $\left.=60.713, \mathrm{df}=6, p=3.224 \times 10^{-11}\right)$, nitrites $($ Chi-Square= 128.26, $\left.\mathrm{df}=8, p=2.2 \times 10^{-16}\right)$, silicates (Chi-Square $\left.=81.528, \mathrm{df}=10, p=2.518 \times 10^{-13}\right)$, and phosphates $\left(\right.$ Chi-Square $=76.454, \mathrm{df}=4, p=9.814 \times 10^{-16}$ )

\begin{tabular}{llll}
\hline Explanatory Variable & Chi-Square & DF & $P$ \\
\hline Season & 109.16 & 3 & $2.2 \times 10^{-165}$ \\
Temperature & 145 & 11 & $2.2 \times 10^{-165}$ \\
Salinity & 145 & 11 & $2.2 \times 10^{-165}$ \\
Dissolved Oxygen & 138.19 & 9 & $2.2 \times 10^{-165}$ \\
$\mathrm{pH}$ & 134.6 & 9 & $2.2 \times 10^{-165}$ \\
Nitrates & 60.713 & 6 & $3.224 \times 10^{-115}$ \\
Nitrites & 128.26 & 8 & $2.2 \times 10^{-165}$ \\
Phosphates & 76.454 & 4 & $9.814 \times 10^{-165}$ \\
Silicates & 81.528 & 10 & $2.518 \times 10^{-135}$ \\
\hline
\end{tabular}

Significance codes of $p$ values: $\mathrm{S}=$ significant, $\mathrm{N}=$ not significant

Table 3: Kruskal-Wallis test showing the effect of season, seawater temperature, salinity, dissolved oxygen concentration, nitrates, nitrites, phosphates, and silicates on phytoplankton species richness. The effect of season on species richness was significant (Chi-Square $=99.435, \mathrm{df}=3, p=2.2 \times 10^{-16}$ ), as well as the effect of temperature on the species richness (Chi-Square $=145, \mathrm{df}=11, p=2.2 \times 10^{-16}$ ). The effect of salinity on species richness was also significant $\left(\right.$ Chi-Square $\left.=145, \mathrm{df}=11, p=2.2 \times 10^{-16}\right)$, as well as the effect of dissolved oxygen on species richness $($ Chi-Square $=39.28, \mathrm{df}=8, p=4.368 \mathrm{x}$ $10^{-06}$ ). The effect of $\mathrm{pH}$ was also significant (Chi-Square $=130.65, \mathrm{df}=9, p=2.2 \times 10^{-16}$ ) along with those of nitrates (Chi-Square $=94.832, \mathrm{df}=6, p=2.2 \mathrm{x}$ $\left.10^{-16}\right)$, nitrites (Chi-Square $\left.=125.55, \mathrm{df}=8, p=2.2 \times 10^{-16}\right)$, silicates (Chi-Square $=113.83, \mathrm{df}=10, p=2.2 \times 10^{-16}$ ) and phosphates (Chi-Square $=67.022, \mathrm{df}$ $=4, p=9.645 \times 10^{-14}$ )

\begin{tabular}{llll}
\hline Explanatory Variable & Chi-Square & DF & $P$ \\
\hline Season & 99.435 & 3 & $2.2 \times 10^{-165}$ \\
Temperature & 145 & 11 & $2.2 \times 10^{-165}$ \\
Salinity & 145 & 11 & $2.2 \times 10^{-165}$ \\
Dissolved oxygen & 143.15 & 9 & $2.2 \times 10^{-165}$ \\
pH & 130.65 & 9 & $2.2 \times 10^{-165}$ \\
Nitrates & 94.832 & 6 & $2.2 \times 10^{-165}$ \\
Nitrites & 125.55 & 8 & $2.2 \times 10^{-165}$ \\
Phosphates & 67.022 & 4 & $9.645 \times 10^{-145}$ \\
Silicates & 113.83 & 10 & $2.2 \times 10^{-165}$ \\
\hline
\end{tabular}

Significance codes of $p$ values: $\mathrm{S}=$ significant, $\mathrm{N}=$ not significant

Table 4: Kruskal-Wallis test showing the effect of abundance on species richness and vice versa. The effect of abundance on species richness was significant $\left(\right.$ Chi-Square $\left.=125.04, \mathrm{df}=8, p=2.2 \times 10^{-16}\right)$, as well as the effect of species richness on abundance $\left(\right.$ Chi-Square $\left.=128.31, \mathrm{df}=7, p=2.2 \times 10^{-16}\right)$

\begin{tabular}{llll}
\hline Explanatory Variable & Chi-Square & DF & $P$ \\
\hline Abundance & 125.04 & 8 & $2.2 \times 10^{-165}$ \\
Species Richness & 128.31 & 7 & $2.2 \times 10^{-165}$ \\
\hline
\end{tabular}

Significance codes of $p$ values: $\mathrm{S}=$ significant, $\mathrm{N}=$ not significant

aspects of the water (Buzzi, 2002; Touliabah et al., 2016). Water temperature in the present study was obviously lower in winter $\left(7.2^{\circ} \mathrm{C}\right)$ compared to summer $\left(29.4^{\circ} \mathrm{C}\right)$.

The temperature values recorded during spring and summer appeared to be suitable for phytoplankton growth, where phytoplankton species richness reached its maximum value. These data are in conformity with those of Boyd et al. (2013), who recorded that the optimal temperature for phytoplankton growth is $10-33.2^{\circ} \mathrm{C}$. In the present study, the water around Umm Al Namil Island was alkaline. (Fig. 2D; 3D), where it ranged between 8.2 in autumn and 8.6 in winter. 
Table 5: List of phytoplankton found at each sampling event (month) in this study with total abundance, species richness and individual species abundance. The abundance is estimated as individual per 5 liters (ind $5 \mathrm{I}^{-1}$ ). Table also shows the season that coincided with every month

\begin{tabular}{|c|c|c|c|c|c|c|}
\hline Month. Year & Event & Season & Species & $\begin{array}{l}\text { Individual } \\
\text { abundance } \\
\text { species }\end{array}$ & Group & $\begin{array}{l}\text { Species } \\
\text { richness }\end{array}$ \\
\hline Sept. 2016 & 1 & Summer & $\begin{array}{l}\text { Chaetoceros affinis } \\
\text { Navicula directa } \\
\text { Cyclotella stylorum } \\
\text { Ditylum brightwellii } \\
\text { Biddulphia pulchella } \\
\text { Bacillaria paxillifera } \\
\text { Amphora sulcata } \\
\text { Cylindrotheca closterium } \\
\text { Trichodesmium erythraeum } \\
\text { Ceratium tripos } \\
\text { Noctiluca scintillans }\end{array}$ & $\begin{array}{l}1 \\
2 \\
2 \\
3 \\
3 \\
3 \\
2 \\
2 \\
6 \\
1 \\
2\end{array}$ & $\begin{array}{l}\text { Diatoms } \\
\text { Diatoms } \\
\text { Diatoms } \\
\text { Diatoms } \\
\text { Diatoms } \\
\text { Diatoms } \\
\text { Diatoms } \\
\text { Diatoms } \\
\text { Cyanobacteria } \\
\text { Dinoflagellates } \\
\text { Diatoms }\end{array}$ & $\begin{array}{l}11 \text { Total } \\
\text { abundance } \\
27\end{array}$ \\
\hline Oct. 2016 & 2 & Autumn & $\begin{array}{l}\text { Navicula directa } \\
\text { Nitzschia longissima } \\
\text { Guinardia flaccida } \\
\text { Coscinodiscus centralis } \\
\text { Melosira moniliformis } \\
\text { Bellerochea horologicalis } \\
\text { Lauderia annulata } \\
\text { Thalassiosira eccentrica } \\
\text { Thalassiothrix longissima } \\
\text { Leptocylindrus danicus } \\
\text { Odontella mobiliensis } \\
\text { Gyrosigma diminutum } \\
\text { Ditylum brightwellii } \\
\text { Biddulphia pulchella } \\
\text { Triceratium reticulum } \\
\text { Pseudonitzschia delicatissima } \\
\text { Corethron criophilum } \\
\text { Licmophora abbreviata } \\
\text { Amphora sulcata } \\
\text { Scenedesmus quadricauda } \\
\text { Cylindrotheca closterium } \\
\text { Pediastrum duplex var. clathratum } \\
\text { Trichodesmium erythraeum } \\
\text { Oscillatoria nigroviridis } \\
\text { Ceratium tripos } \\
\text { Noctiluca scintillans }\end{array}$ & $\begin{array}{l}3 \\
2 \\
3 \\
2 \\
2 \\
2 \\
1 \\
3 \\
1 \\
3 \\
3 \\
1 \\
1 \\
3 \\
2 \\
1 \\
1 \\
2 \\
1 \\
2 \\
1 \\
2 \\
1 \\
3 \\
1 \\
3\end{array}$ & $\begin{array}{l}\text { Diatoms } \\
\text { Diatoms } \\
\text { Diatoms } \\
\text { Diatoms } \\
\text { Diatoms } \\
\text { Diatoms } \\
\text { Diatoms } \\
\text { Diatoms } \\
\text { Diatoms } \\
\text { Diatoms } \\
\text { Diatoms } \\
\text { Diatoms } \\
\text { Diatoms } \\
\text { Diatoms } \\
\text { Diatoms } \\
\text { Diatoms } \\
\text { Diatoms } \\
\text { Diatoms } \\
\text { Diatoms } \\
\text { Diatoms } \\
\text { Diatoms } \\
\text { Diatoms } \\
\text { Cyanobacteria } \\
\text { Cyanobacteria } \\
\text { Dinoflagellates } \\
\text { Dinoflagellates }\end{array}$ & $\begin{array}{l}\text { Species richness } \\
26 \text { Total } \\
\text { abundance } \\
50\end{array}$ \\
\hline Nov. 2016 & 3 & Autumn & $\begin{array}{l}\text { Navicula directa } \\
\text { Guinardia flaccida } \\
\text { Cyclotella stylorum } \\
\text { Bellerochea horologicalis } \\
\text { Lauderia annulata } \\
\text { Thalassiosira eccentrica } \\
\text { Thalassiothrix longissima } \\
\text { Leptocylindrus danicus } \\
\text { Odontella mobiliensis } \\
\text { Pleurosigma elongatum } \\
\text { Fragilaria martyi } \\
\text { Ditylum brightwellii } \\
\text { Bacillaria paxillifera } \\
\text { Triceratium reticulum } \\
\text { Corethron criophilum }\end{array}$ & $\begin{array}{l}2 \\
3 \\
2 \\
2 \\
1 \\
5 \\
2 \\
2 \\
2 \\
2 \\
2 \\
1 \\
2 \\
2 \\
2\end{array}$ & $\begin{array}{l}\text { Diatoms } \\
\text { Diatoms } \\
\text { Diatoms } \\
\text { Diatoms } \\
\text { Diatoms } \\
\text { Diatoms } \\
\text { Diatoms } \\
\text { Diatoms } \\
\text { Diatoms } \\
\text { Diatoms } \\
\text { Diatoms } \\
\text { Diatoms } \\
\text { Diatoms } \\
\text { Diatoms } \\
\text { Diatoms }\end{array}$ & $\begin{array}{l}\text { Species richness } \\
23 \text { Total } \\
\text { abundance } \\
47\end{array}$ \\
\hline
\end{tabular}




\begin{tabular}{|c|c|c|c|c|c|c|}
\hline & & & Skeletonema costatum & 1 & Diatoms & \\
\hline & & & Licmophora abbreviata & 1 & Diatoms & \\
\hline & & & Scenedesmus quadricauda & 1 & Diatoms & \\
\hline & & & Cylindrotheca closterium & 2 & Diatoms & \\
\hline & & & Pediastrum duplex var. clathratum & 3 & Diatoms & \\
\hline & & & Trichodesmium erythraeum & 2 & Cyanobacteria & \\
\hline & & & Oscillatoria nigroviridis & 2 & Cyanobacteria & \\
\hline & & & Ceratium tripos & 3 & Dinoflagellates & \\
\hline \multirow[t]{7}{*}{ Dec. 2016} & 4 & Winter & Guinardia flaccida & 5 & Diatoms & Species richness \\
\hline & & & Bellerochea horologicalis & 2 & Diatoms & 7 Total \\
\hline & & & Thalassionema nitzschioides & 6 & Diatoms & abundance \\
\hline & & & Trichodesmium erythraeum & 2 & Diatoms & 20 \\
\hline & & & Lyngbya majuscula & 1 & Cyanobacteria & \\
\hline & & & Protoperidinium murrayi & 1 & Dinoflagellates & \\
\hline & & & Dinophysis caudata & 3 & Dinoflagellates & \\
\hline \multirow[t]{11}{*}{ Jan. 2017} & 5 & Winter & Navicula directa & 2 & Diatoms & Species richness \\
\hline & & & Nitzchia longissima & 2 & Diatoms & 11 Total \\
\hline & & & Cyclotella stylorum & 1 & Diatoms & abundance \\
\hline & & & Bellerochea horologicalis & 2 & Diatoms & 19 \\
\hline & & & Thalassionema nitzschioides & 1 & Diatoms & \\
\hline & & & Pleurosigma elongatum & 1 & Diatoms & \\
\hline & & & Ditylum brightwellii & 1 & Diatoms & \\
\hline & & & Trichodesmium erythraeum & 3 & Cyanobacteria & \\
\hline & & & Protoperidinium murrayi & 2 & Dinoflagellates & \\
\hline & & & Ceratium tripos & 2 & Dinoflagellates & \\
\hline & & & Gymnodinium gracilentum & 2 & Dinoflagellates & \\
\hline \multirow[t]{12}{*}{ Feb. 2017} & 6 & Winter & Bacteriastrum delicatulum & 1 & Diatoms & Species richness \\
\hline & & & Navicula directa & 4 & Diatoms & 11 Total \\
\hline & & & Guinardia flaccida & 1 & Diatoms & abundance \\
\hline & & & Bellerochea horologicalis & 3 & Diatoms & 25 \\
\hline & & & Thalassiosira eccentrica & 3 & Diatoms & \\
\hline & & & Biddulphia pulchella & 1 & Diatoms & \\
\hline & & & Hemidiscus cuneiformis & 1 & Diatoms & \\
\hline & & & Amphiprora plicata & 1 & Diatoms & \\
\hline & & & Amphora sulcata & 2 & Diatoms & \\
\hline & & & Hemiaulus sinensis & 3 & Diatoms & \\
\hline & & & Trichodesmium erythraeum & 2 & Cyanobacteria & \\
\hline & & & Protoperidinium murrayi & 3 & Diatoms & \\
\hline \multirow[t]{12}{*}{ Mar. 2017} & 7 & Spring & Chaetoceros affinis & 1 & Diatoms & Species richness \\
\hline & & & Navicula directa & 2 & Diatoms & 11 Total \\
\hline & & & Pseudonitzschia delicatissima & 2 & Diatoms & abundance \\
\hline & & & Ditylum brightwellii & 2 & Diatoms & 25 \\
\hline & & & Biddulphia pulchella & 3 & Diatoms & \\
\hline & & & Bacillaria paxillifera & 4 & Diatoms & \\
\hline & & & Amphora sulcata & 2 & Diatoms & \\
\hline & & & Hemiaulus sinensis & 4 & Diatoms & \\
\hline & & & Trichodesmium erythraeum & 1 & Cyanobacteria & \\
\hline & & & Ceratium tripos & 2 & Dinoflagellates & \\
\hline & & & Dinophysis caudata & 1 & Dinoflagellates & \\
\hline & & & Prorocentrum micans & 1 & Dinoflagellates & \\
\hline \multirow[t]{8}{*}{ Apr. 2017} & 8 & Spring & Bacteriastrum delicatulum & 2 & Diatoms & Species richness \\
\hline & & & Coscinodiscus centralis & 3 & Diatoms & 11 Total \\
\hline & & & Thalassionema nitzschioides & 2 & Diatoms & abundance \\
\hline & & & Pseudonitzschia delicatissima & 2 & Diatoms & 28 \\
\hline & & & Asterionellopsis glacialis & 2 & Diatoms & \\
\hline & & & Licmophora abbreviata & 4 & Diatoms & \\
\hline & & & Amphora sulcata & 2 & Diatoms & \\
\hline & & & Trichodesmium erythraeum & 7 & Cyanobacteria & \\
\hline
\end{tabular}




\begin{tabular}{|c|c|c|c|c|c|c|}
\hline & & & Ceratium tripos & 2 & Dinoflagellates & \\
\hline & & & Dinophysis caudata & 2 & Dinoflagellates & \\
\hline \multirow[t]{13}{*}{ May. 2017} & 9 & Summer & Chaetoceros affinis & 1 & Diatoms & Species richness \\
\hline & & & Pseudonitzschia delicatissima & 1 & Diatoms & 13 Total \\
\hline & & & Thalassiosira eccentrica & 1 & Diatoms & abundance \\
\hline & & & Bacillaria paxillifera & 1 & Diatoms & 20 \\
\hline & & & Asrerionellopsis glacialis & 3 & Diatoms & \\
\hline & & & Planktoniella sol & 1 & Diatoms & \\
\hline & & & Diploneis weissflogii & 3 & Diatoms & \\
\hline & & & Amphora sulcata & 1 & Diatoms & \\
\hline & & & Pediastrum duplex var. clathratum & 1 & Cyanobacteria & \\
\hline & & & Oscillatoria nigroviridis & 2 & Cyanobacteria & \\
\hline & & & Lyngbya majuscula & 2 & Diatoms & \\
\hline & & & Ceratium tripos & 2 & Diatoms & \\
\hline & & & Prorocentrum micans & 1 & Diatoms & \\
\hline \multirow[t]{10}{*}{ Jun. 2018} & 10 & Summer & Cyclotella stylorum & 1 & Diatoms & Species richness \\
\hline & & & Thalassiosira eccentrica & 1 & Diatoms & 10 Total \\
\hline & & & Bacillaria paxillifera & 3 & Diatoms & abundance \\
\hline & & & Asrerionellopsis glacialis & 4 & Diatoms & 26 \\
\hline & & & Diploneis weissflogii & 4 & Diatoms & \\
\hline & & & Dunaliella bioculata & 1 & Chlorophyta & \\
\hline & & & Oscillatoria nigroviridis & 5 & Cyanobacteria & \\
\hline & & & Lyngbya majuscula & 4 & Cyanobacteria & \\
\hline & & & Ceratium tripos & 1 & Dinoflagellates & \\
\hline & & & Prorocentrum micans & 2 & Dinoflagellates & \\
\hline \multirow[t]{7}{*}{ Jul. 2018} & 11 & Summer & Cyclotella stylorum & 2 & Diatoms & Species richness \\
\hline & & & Thalassiosira eccentrica & 3 & Diatoms & 7 Total \\
\hline & & & Asrerionellopsis glacialis & 2 & Diatoms & abundance \\
\hline & & & Diploneis weissflogii & 3 & Diatoms & 19 \\
\hline & & & Dunaliella bioculata & 1 & Chlorophyta & \\
\hline & & & Lyngbya majuscula & 5 & Cyanobacteria & \\
\hline & & & Protoperidinium murrayi & 3 & Dinoflagellates & \\
\hline \multirow[t]{4}{*}{ Aug. 2017} & 12 & Summer & Diploneis weissflogii. & 5 & Diatoms & Species richness \\
\hline & & & Dunaliella bioculata & 1 & Chlorophyta & 4 Total \\
\hline & & & Lyngbya majuscula & 4 & Cyanobacteria & abundance \\
\hline & & & Dinophysis caudata & 3 & Dinoflagellates & \\
\hline
\end{tabular}

These $\mathrm{pH}$ values are suitable for high phytoplankton standing crop, which produced highest value in the same season where diatoms, dinoflagellates and blue-green algal species showed maximum growth above $\mathrm{pH} 8$. Similar observations were also reported by Touliabah et al. (2010) and El Gammal (2017) in the coastal waters of Saudi Arabia. Such high pH values in this study could be due to higher photosynthetic consumption of carbon dioxide (Hinga, 2002).

The DO behavior in this study being higher during autumn, summer, and spring compared to winter as explained earlier may be due to increased SST and breakdown of planktonic organisms, organic matter, and detritus where oxygen is consumed and carbon dioxide is released. This shows that oxygen solubility decreases with rising salinity and temperature (Calliari et al., 2005). Wide inconstancies in temperature were observed between winter and summer. The wide-ranging seasonal discrepancy in temperature in coastal water (Abdul Azis et al., 2003) is considerably an impact of stern weather of the study area and strident condition of the Gulf region (Price, 1979). Arabian Gulf is a semi-enclosed system where evaporation is one of the most valuable and substantial factor that changes the salinity. There was no such huge variation in salinity, that exhibits the highest value (47.1 psu) during summer and the lowest values (42.0 psu) during autumn (Fig. 2C). Higher salinity values during summer may be associated to excessive evaporation rate as stated by Khomayis (2002) and Schumann et al. (2006). In the present study (Table 3), the effect of salinity on species richness was significant. Schumann et al. (2006) demonstrated that salinity affects the phytoplankton diversity. It has been reported that increasing salinity rises phytoplankton diversity but lowers both phytoplankton biomass and production further, Polikarpov et al. (2008). Thus, more studies have to be conducted near Umm Al-Namil Island in future.

The present investigation data revealed that the lowest phosphate concentration $\left(0.19 \mathrm{mg} \mathrm{kg}{ }^{-1}\right)$ was observed during summer, while the highest during autumn $\left(0.7 \mathrm{mg} \mathrm{kg}^{-1}\right.$; Fig. $\left.2 \mathrm{~F}\right)$. 
○

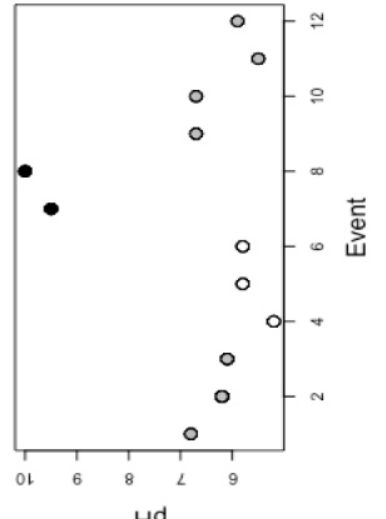

$\mathrm{Hd}^{\mathrm{d}}$

0

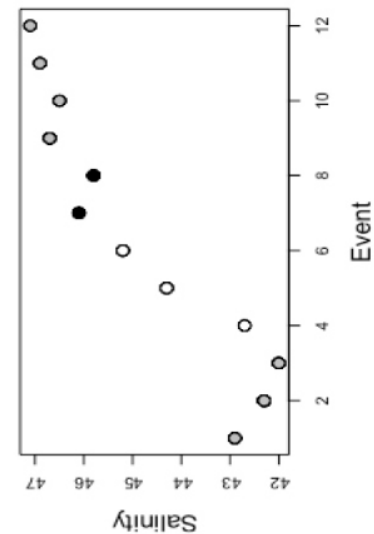

$\infty$

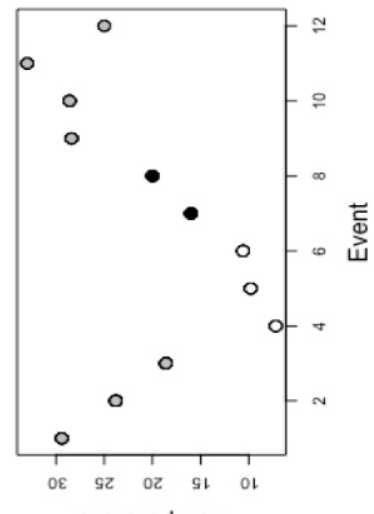

әฺกฺฺอ

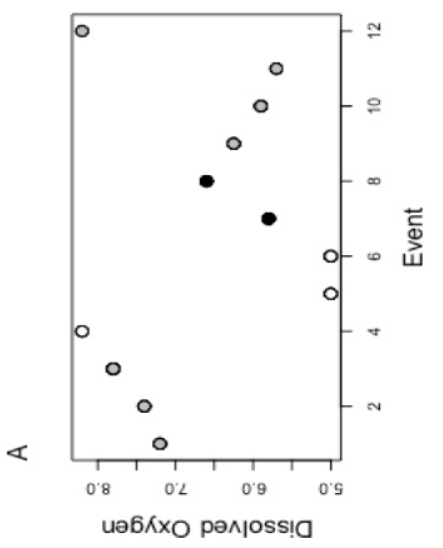

I

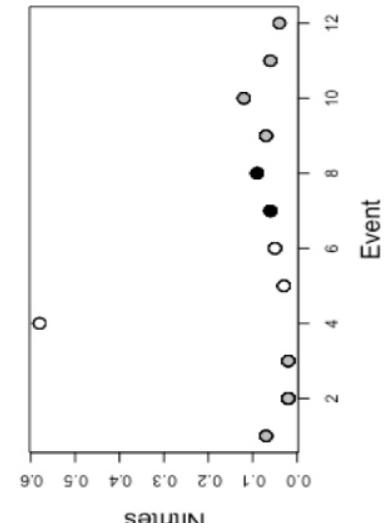

sel!!ฺ!N

0

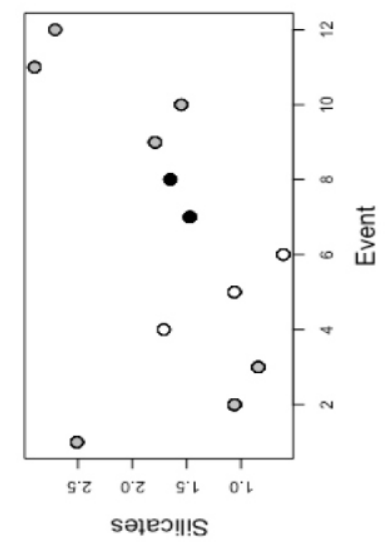

4

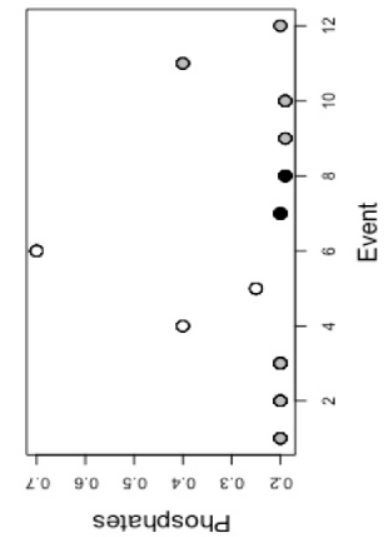

ш

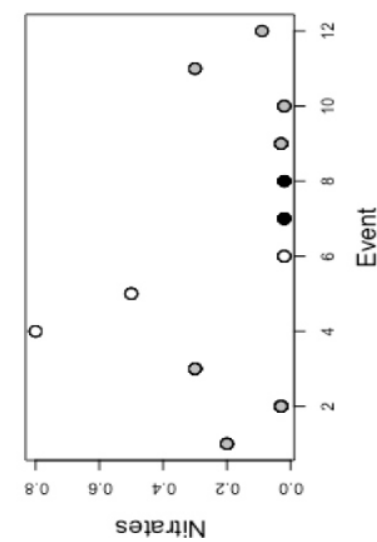

올

$\stackrel{\div}{\frac{1}{0}}$

हृ

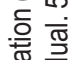

능

엉 잉

ฮิ ฐ

종

क

Oफ

这은

흠

$\frac{\sqrt{0}}{0} \stackrel{-}{=}$

言

닌

记

궁

혼

需

웜 함

음 틍ㅁㅁ

क 웡

Ð ․ㅓ․ 음

등

๘ $\widehat{0}$

흔

오흫

응 읕듬

क

중

ब)

들듬 흔

잉만단

는

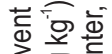

屯

을 है 임

产苋

क

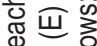

호동ㅎㅇ

क⿻

ฮับ

实

ॠ

정 는

응 䏯

응 $\frac{\mathrm{O}}{\mathrm{d}}$

등

즁ㅇㅇㅇ

ह

둔 $\frac{\pi}{0}$

ờ है

क्ज

这

iv

ㅎํ ๗ 
A

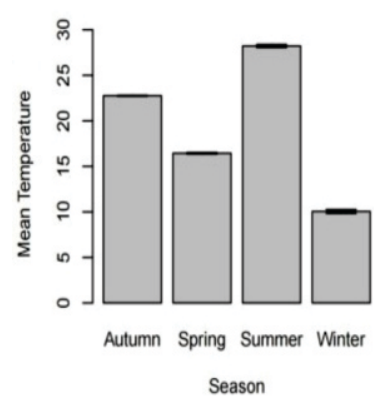

$\mathrm{E}$
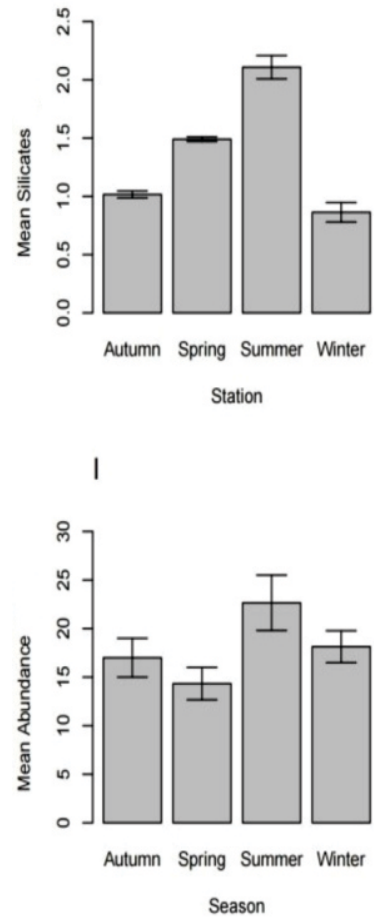

B

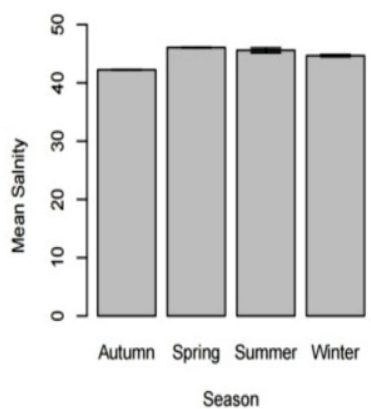

$\mathrm{F}$

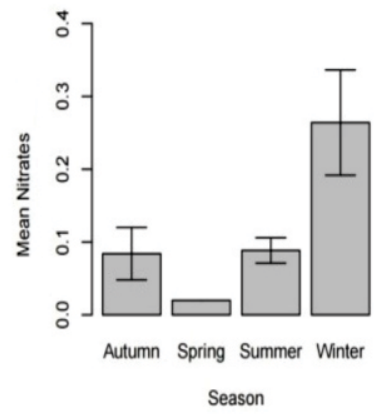

$J$

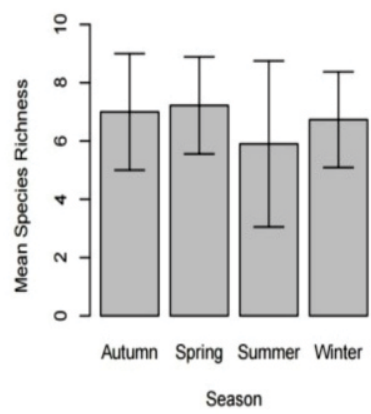

C

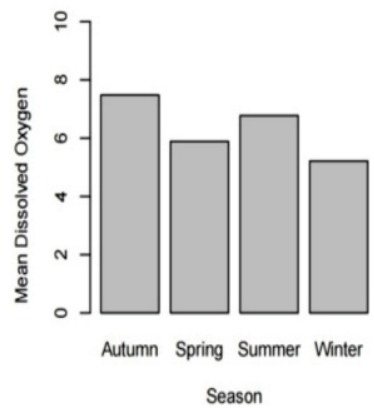

G

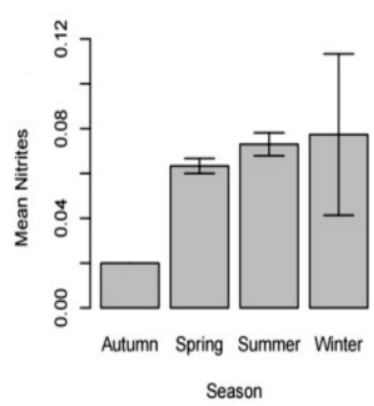

D

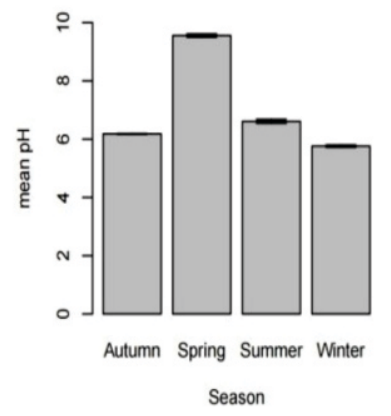

$\mathrm{H}$

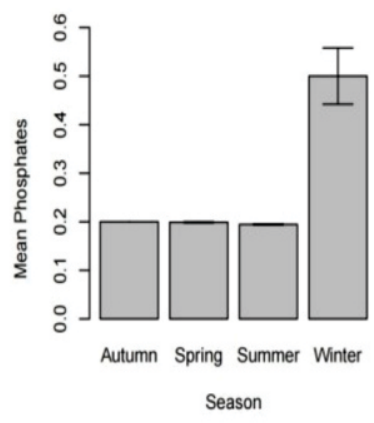

Fig. 3: Mean values with standard error bars for physico-chemical and biological parameters at each season in the subtidal water of Umm Al-Namil Island. (A) Seawater temperature $\left({ }^{\circ} \mathrm{C}\right) ;(\mathrm{B})$ Salinity (PSU); (C) Dissolved oxygen concentration ( $\left.\mathrm{mg} \mathrm{I}^{-1}\right) ;(\mathrm{D}) \mathrm{pH}$; (E) Silicates $\left(\mathrm{mg} \mathrm{kg}^{-1}\right) ;(\mathrm{F})$ Nitrates $\left(\mathrm{mg} \mathrm{kg}^{-1}\right) ;(\mathrm{G})$ Nitrites $\left(\mathrm{mg} \mathrm{kg}^{-1}\right) ;(\mathrm{H})$ Phosphates $\left(\mathrm{mg} \mathrm{kg}^{-1}\right)$; (I)Abundance (individual. $5 \mathrm{I}^{-1}$ ) and $(\mathrm{J})$ Species richness (S). Unseen error bars due to small variation in data.

This could be related to reduced uptake rates by phytoplankton in summer than autumn; this was confirmed by the reduced phytoplankton standing crop recorded during summer compared with autumn where phosphate reached its lowest value (Touliabah et al., 2016). Nitrite $\left(0.58 \mathrm{mg} \mathrm{kg}^{-1}\right)$ and silicate $(0.95 \mathrm{mg}$ $\mathrm{kg}^{-1}$ ) attained their maximum values in winter and autumn, while the minimum value $\left(0.02 \mathrm{mg} \mathrm{kg}^{-1}\right.$ and $\left.0.61 \mathrm{mg} \mathrm{kg}^{-1}\right)$ was observed during winters for both nitrite and silicate, respectively. Nitrate reached its maximum value $\left(0.26 \mathrm{mg} \mathrm{kg}^{-1}\right)$ during spring, and minimum $\left(0.16 \mathrm{mg} \mathrm{kg}^{-1}\right)$ during winter and summer with similar values. In this study, higher levels of nitrite, nitrate and silicates were observed due to discharge of domestic waste waters in around the area. Normally, nitrogen and phosphorous are present in the domestic waste waters (El-Bassat and Taylor, 2007), hence, presence of both are presumed in the waters of study area. A total of 46 species belonging to 4 groups were identified from the water of Umm Al-Namil Island. Bacillariophyceae were frequently present (72\%) comprising Dinophyceae, Cyanophyceae and Chlorophyceae (13\%, 7\% and $8 \%$ ) respectively. The most abundant phytoplankton was observed during October (autumn), while minimum during August (summer). This was also observed by Abdulaziz et al. (2000) in the coastal waters of Saudi Arabia. During autumn, phytoplankton peaks were primarily due to the presence of Dinophyceae, 
A

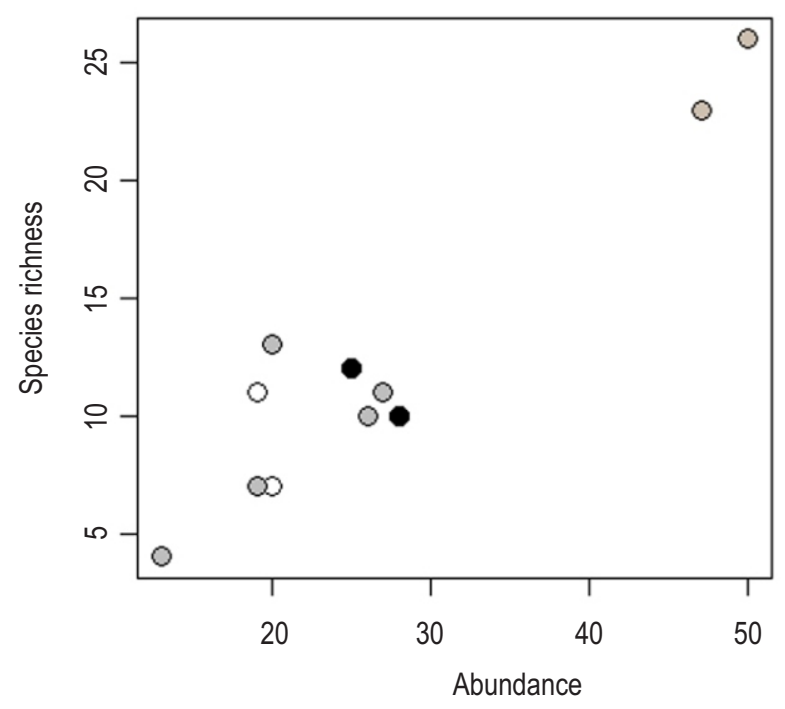

B

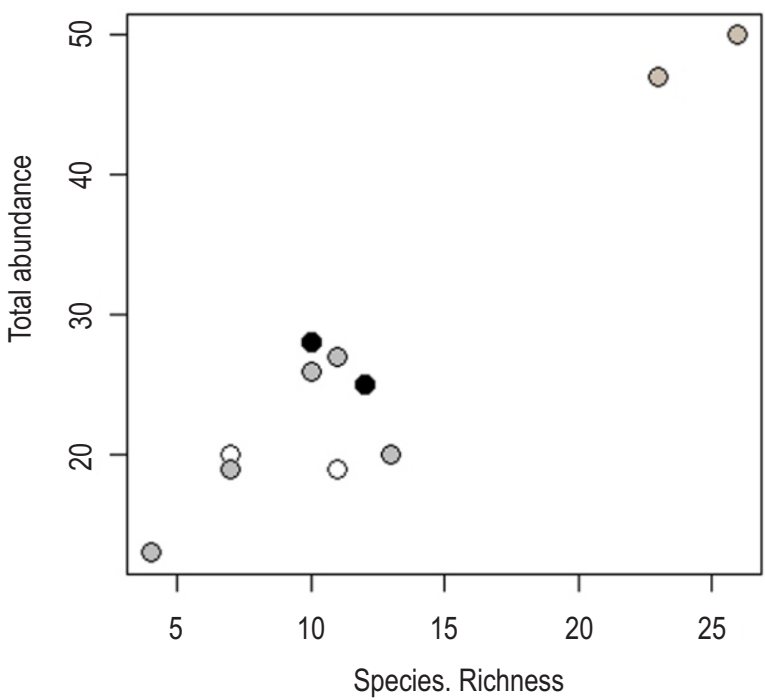

Fig. 4: PlotA shows a direct relation between species richness and abundance and opposite is also true as shown in plot $B$. Colors represent seasons as follows: Antique white for autumn, black for spring, grey for summer, and dark grey for winter. Such clear relationship in plots A and B appeared in all seasons. Table 4 explains the relationship in statistical terms.

Cyanophyceae and Chlorophyceae. Similar results were also observed by Turkoglu (2010). The phytoplankton community composition in this were study showed high abundance values, although species richness was low. This may be associated with the existence of some stressors that have restricted the species diversity to only lenient and permissive species to these particular environmental circumstances (Parnell, 2003). Diatoms were dominant in the subtidal waters of Umm Al-Namil Island followed by dinoflagellates (Table 5). This converse with the numerical dominance of diatoms in species number reported in other phytoplankton composition studies done at tropical latitudes (Rojas-Herrera et al., 2012 b; Moreno-Díaz et al., 2015; VaronaCordero et al., 2014; Rojas-Herrera et al., 2012a,b). The groups of phytoplankton species are considered good relevance within marine environment water masses (Castillo et al., 1993). During the course of survey, Dinophyceae sustained as fully evolved assemblage during winter.

Thirty-three species associated to Bacillariophyceae were analyzed. The highest abundance of Bacillariophyceae was detected in autumn and winter. The dominant species of Bacillariophyceae in most events were Thalassiosira eccentrica, Navicula directa, Amphora sulcata, Bacillaria paxillifera, Bellerochea horologicalis, Cyclotella stylorum, Ditylum brightwellii, Diploneis weissflogii, Guinardia flaccida, Asterionellopsis glacialis and Biddulphia pulchella. High quantity of nutrient in sewage water was primary disputation for the diatoms to grow successfully during this period. Varela and Prego (2003) also reported the dominance of diatoms in the Coruna Harbour (North West of Spain) to the nutrient loaded seawater.
Morais et al. (2003) stated that the increase in water temperature might have allowed phytoplankton to optimize its growth and productivity. Subsequently, Dinophyceae was also dominant in the phytoplankton community after diatoms. Amongst this, the most predominant species in the study site were Ceratium tripos, Dinophysis caudata and Protoperidinium murrayi. Cyanophyceae was the third prevalent group during the present investigation, constituting 7\% of the total phytoplankton composition. Three species recorded in this research, were Trichodesmium erythraeum, Lyngbya majuscula and Oscillatoria nigro viridis. Chlorophyceae were infrequently documented and this result concurs with the reports of (2005) from the South China Sea. The presence of Chlorophyceae was restricted to few sampling events, the dominant species were Pediastrum duplex var. clathratum, Cylindrotheca closterium, Dunaliella bioculata and Scenedesmus quadricauda.

Diatoms Navicula directa, Guinardia flaccida and Thalassiosira eccentrica dominated during autumn (OctoberNovember 2016), and early dry season (Table 5). While dinoflagellate Noctiluca scintillans and Cyanophytes Trichodesmium erythraeum and Oscillatoria nigroviridis dominated only during the autumn season. Some studies indicate that changes in phytoplankton community structure can be conjoined with small changes in the sea surface temprature or nutrients concentrations in the water column (Langlois and Smith, 2001). The decrease in water temperature from November 2016 to March 2017 may have affected dinoflagellate abundance during this period as this microalgal group is highly sensitive to minor fluctuations in the temperature (Rojas-Herrera et al., 2012; 


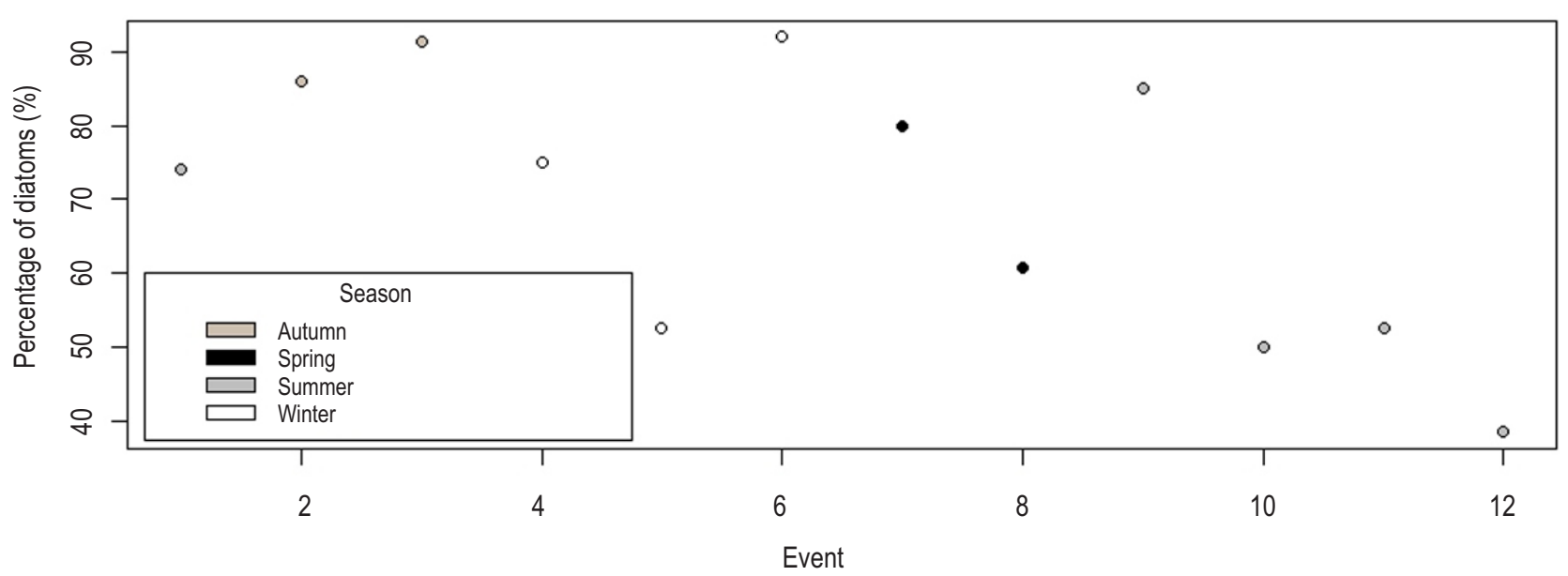

Fig. 5: Percentage of diatoms among other phytoplankton in the samples of each event (i.e., month).The maximum percentage was $92 \%$ at event 6 , while the lowest percentage (38.46\%) was observed at event 12 . Each color represents a season as shown on the legend.

Varona- Cordero et al., 2014; Moreno-Díaz et al., 2015). Diatoms, in disparity, are less sensitive to slight shifts in water temperature and responds more speedily to changing nutrient concentrations (Örnólfsdóttir et al., 2004). Dominance of diatoms can be attributed to the presence of higher relative abundance of Navicula directa, Guinardia flaccida, Thalassiosira eccentrica, Biddulphia pulchella, Bacillaria paxillifera and Diploneis weiflogii (Table 5) during this study period. Among the six dinoflagellate species identified here, at least one (Gymnodinium gracilentum) has been reported to cause harmful algal blooms (HABs) in Kuwait waters (Al-Yamani et al., 2000, 2012). In the present case, however, the majority of dinoflagellates like Protoperidinium murrayi, Ceratium tripos, Noctiluca scintillans, Dinophysis caudata and Prorocentrum micans found were not toxic (Table 5).

Species richness ( 46 species) and abundance values (13 to 50 ind $\left.5 l^{-1}\right)$ recorded in the study area were within ranges previously stated in the other countries with similar climatic conditions (Rojas-Herrera etal., 2012 b; Moreno-Díaz et al., 2015; Rojas-Herrera et al., 2012). The low values observed in this study commend that species composition alter among months in counter to fluctuation in environmental conditions over time. Inclusive, the results indicate that phytoplankton species richness and species abundance in Umm Al-Namil Island varied temporally in response to inconstancy in environmental conditions. Primary among these variations were higher nutrient concentrations and high discrepancy in water temperature in different seasons of the year contributed population growth in some dinoflagellate or diatom species at different times during the year. Furthermore, in another study in the same study area herein, the high species richness and abundance of phytoplankton observed seem to had reflected on the planktonic food web, in which nine larval fish families were recorded (Acropomatidae, Bregmacerotidae, Bythitidae, Clupeidae,
Engraulidae, Leioganthidae, Platycephalidae, Pseudochromidae and Sparidae) along with other zooplankton, mainly Copepoda, Mollusca larvae and Radiolaria (Ali et al., 2020). In addition, it has been reported that diatoms are among the most vulnerable (mainly consumed) species in the Arabian Gulf food web (Ali, 2015). Diatoms are greater habituated to coastal environments, while dinoflagellates are better preferred to the oceanic environment (Castillo et al., 1993; Rojas-Herrera et al., 2012 b). The presence of diversity in the monthly cell profusion of these two substantial microalgal groups during the entire year (Fig. 31, Table 5), propose that environmental circumstances in Umm AlNamil Island's subtidal waters alter during the year in feedback to variations in numerous physico-chemical parameters. Alteration in the Kuwait Bay's water masses are supposedly echoed in the changing nature of its phytoplankton community. Different species prevail numerically during each month, signifying extensive variability in species composition in the course of time. Kruskal-Wallis test showed that the effect of temperature on phytoplankton abundance (Chi-Square $=145, \mathrm{df}=11, p=2.2 \times 10$. ${ }^{16}$ ) salinity, $\left(\right.$ Chi-Square $\left.=145, \mathrm{df}=11, p=2.2 \times 10^{-16}\right)$ and DO (ChiSquare $\left.=138.19, \mathrm{df}=9, p=2.2 \times 10^{-16}\right)$ were significant $($ Table 2). Likewise, $\mathrm{pH}$ (Chi-Square $=134.6, \mathrm{df}=9, p=2.2 \times 10^{-16}$ ), various nutrients (Chi-Square $=60.713, \mathrm{df}=6, p=3.224 \times 10^{-11}$ ), nitrites (Chi-Square $=128.26, \mathrm{df}=8, p=2.2 \times 10^{-16}$ ), silicates (ChiSquare $=81.528, \mathrm{df}=10, p=2.518 \times 10^{-13}$ ) and phosphates (ChiSquare $\left.=76.454, \mathrm{df}=4, p=9.814 \times 10^{-16}\right)$ had significant effect on phytoplankton abundance. (Table 2). Season also had a significant effect on the abundance of phytoplankton species (Chi-Square $\left.=109.16, \mathrm{df}=3, p=2.2 \times 10^{-16}\right)($ Table 2).

Similarly, a significant effect of season (Chi-Square = 99.435, $\mathrm{df}=3, p=2.2 \times 10^{-16}$ ) and temperature was observed on the species richness (Chi-Square $=145, \mathrm{df}=11, p=2.2 \times 10^{-16}$ ) (Table 3). The effect of salinity and dissolved oxygen on species richness was also significant (Table 3). The effect of pH, nitrates, 
silicate and phosphate on species richness was also significant (Table 3). The abundance showing statistically significant effect on the species richness in this study irrespective of season with a direct relationship (Chi-Square $=125.04, \mathrm{df}=8, p=2.2 \times 10^{-16}$ ) (Table 4; Fig. 3A). Surprisingly, the other way was also true and species richness had a significant effect on the abundance (ChiSquare $=128.31, \mathrm{df}=7, p=2.2 \times 10^{-16}$ ) (Table 4; Fig. 3B). The phytoplankton assemblages obtained from different temporal gradients over one-year study period in the Umm Al-Namil island's subtidal waters showed a total of 33 diatoms, 6 dinoflagellates, 3 cyanobacteria and 4 chlorophytes. Generally, diatoms have dominated all samples, while the other phytoplankton groups were minor at almost all the events (Table 4; Fig. 5). The highest diatom percentage (over 70\%) was observed at event 6 in winter $(92 \%)$, event 3 in autumn (91.49\%), event 2 in autumn (86\%), event 9 in summer $(85 \%)$, event 7 in spring (80\%), event 4 in winter $(75 \%)$, and event 1 in summer $(74.07 \%$, Fig. 5). Percentage of diatoms was $60.71 \%$ at event 8 in spring (Fig. 5). Percentage of diatoms ranged between 50 to $52.63 \%$ during events 10 (summer), 5 (winter) and 11 (summer), respectively, as the percentage at the latter 2 events are same (Fig. 4). The lowest percentage of diatoms was observed at event 12 in summer (38.46\%) (Fig. 4) that also coincided with lowest abundance and lowest species richness as explained above (Fig. 2J). Other observed phytoplankton groups were dinoflagellates, cyanobacteria and chlorophytes (Table 5). Almost similar observations were reported by from (Chakraborty et al., (2015) from Andaman Sea and Indian Ocean.

The most encountered diatoms Thalassiosira eccentrica, Navicula directa and Amphora sulcata; were present at 6 sampling events at almost all the seasons despite their level of abundance. The abundance of Thalassiosira eccentrica was $3,5,3,1,1,3$ (ind $5 l^{-1}$ ) at events $2,3,6,9,10$ and 11. The abundance of Navicula directa was $2,3,2,2,4,2$ (ind $5 l^{-1}$ ) at events 1,2,3,5,6 and 7. The abundance of Amphora sulcata was 2,1,2,2,2,1 (ind $5 l^{-1}$ ) at events 1,2,6,7,8 and 9, respectively. Diatoms Bacillaria paxillifera, Bellerochea horologicalis, Cyclotella stylorum and Ditylum brightwellii were encountered at 5 sampling events at almost all seasons despite the level of abundance. The abundance of Bacillaria paxillifera was $3,2,4,1,3$ (ind $5 l^{-1}$ ) at events $1,3,7,9$ and 10 . The abundance of Bellerochea horologicalis was 2,2,2,2,3 (ind $5 l^{-1}$ ) at events 2,3,4,5 and 6, (Table 5). The abundance of Cyclotella stylorum was 2,2,1,1,2 (ind $5 l^{-1}$ ) at events 1,3,5,10, and 11, (Table 5). The abundance Ditylum brightwellii was $3,1,1,1,2$ (ind $\left.\left.5\right|^{-1}\right)$, at events 1,2,3,5 and 7, respectively (Table 5).

The diatoms Diploneis weissflogii, Guinardia flaccida, Asterionellopsis glacialis and Biddulphia pulchella were encountered at 4 sampling events at almost all seasons despite the level of abundance. The abundance of Diploneis weissflogii was $3,4,3,5$ (ind $5 l^{-1}$ ) at events $9,10,11$ and 12 . The abundance of Guinardia flaccid was $3,3,5,1$ (ind $51^{-1}$ ) at events $2,3,4$ and 6 . The abundance of Asterionellopsis glacialis was 2,3,4,2 (ind $51^{-1}$ ) at events 8,9,10 and 11. The abundance of Biddulphia pulchella was $3,3,1,3$ (ind $\left.5\right|^{-1}$ ) at events $1,2,6$ and 7 . Diatoms Chaetoceros affinis, Thalassionema nitzschioides and Licmophora abbreviata were encountered at 3 events, irrespective of season (Table 5). The abundance of Chaetoceros affinis was 1,1,1 (ind $51^{-1}$ ) at events 1,7, and 9. The abundance of Thalassionema nitzschioides was 6,1,2 (ind $5 l^{-1}$ ) at events 4,5, and 8, (Table 5). The abundance of Licmophora abbreviata was 2,1,4 (ind $5 l^{-1}$ ) at events 2,3, and 8, respectively. The remaining diatoms appeared in only 2 events irrespective of season (Bacteriastrum delicatulum, Nitzchia longissimi, Coscinodiscus centralis, Lauderia annulata, Thalassiothrix longissima, Leptocylindrus danicus, Odontella mobiliensis, Pleurosigma elongatum, Triceratium reticulum, Pseudonitzschia delicatissima, Corethron criophilum, and Hemiaulus sinensis), or even one event (Melosira moniliformis, Gyrosigma diminutum, Fragilaria martyi, Hemidiscus cuneiformis, Planktoniella sol and Amphipro raplicata) (Table 5).

Dinoflagellates were the second most abundant group of phytoplankton in this study. Only 6 species viz., Ceratium tripos, Dinophysis caudata, Protoperidinium murrayi, Noctiluca scintillans, Prorocentrum micans and Gymnodinium gracilentum were encountered. The most commonly encountered dinoflagellate was Ceratium tripos, which was observed at eight events irrespective of the seasons. The abundance of Ceratium tripos was 1, 1,3,2,2,2,2,1 (ind $\left.5\right|^{-1}$ ), at events 1,2,3,5,7,8,9 and 10. Both Protoperidinium murrayi and Dinophysis caudata were observed only at four events despite seasons. The abundance of Dinophysis caudata was 3,1,2,3 (ind $\left.5\right|^{-1}$ ) at events 4, 7, 8 and 12, while the abundance of Protoperidinium murrayi was $1,2,3,3$ (ind $5 l^{-1}$ ) at events $4,5,6$, and 11 . The abundance of Prorocentrum micans was $1,1,2$ (ind $5 l^{-1}$ ) at events 7,9 and 10, respectively. The abundance Noctiluca scintillans was 2,3 (ind $\left.5\right|^{-1}$ ) at events 1 and 2. The toxic dinoflagellate, Gymnodinium gracilentum was encountered only at event 5 with the abundance value of 2 (ind $\left.5\right|^{-1}$ ) (Table 5).

Chlorophytes were represented in this study by the presence of Pediastrum duplex var. clathratum, Cylindrotheca closterium, Dunaliella bioculata and Scenedesmus quadricauda. The abundance of Pediastrum duplex var. clathratum was 2,3,1 (ind $5 l^{-1}$ ), at events 2,3,9. The abundance of Cylindrotheca closterium was $1,1,2$ (ind $5 l^{-1}$ ), at events 1,2 , and 3.The abundance of Dunaliella bioculata was $1,1,1$ (ind $\left.5\right|^{-1}$ ), at events 10,11 and 12. The abundance of Scenedesmus quadricauda was 2,1 (ind $51^{-1}$ ), at events 2 and 12. Cyanobacteria were represented in this study by Trichodesmium erythraeum, Lyngbya majuscula and Oscillatoria nigro viridis. The most encountered cyanobacteria was Trichodesmium erythraeum at events 1 to 8 covering all seasons, with the abundance values of 1,1,2,2,3,2,1, and 7 (ind $\left.5\right|^{-1}$ ). The abundance of Lyngbya majuscula was $1,2,4,5,2$ (ind $5 l^{-1}$ ), at events 4,9,10,11 and 12. The abundance of Oscillatoria nigro viridis was 2, 2, 2, 5 (ind $\left.5\right|^{-1}$ ), at events 2,3,9 and 10, respectively (Table 5).

The correlation between diversity, species richness, abundance, and their dependence on resource possibility and disproportion have been investigated from divergent standpoint, including the Resource-Ratio Theory (RRT, Tilman 1982), 
Species-Energy Theory (SET, Wright 1983) and the biodiversity ecosystem functioning (BEF) criterion (Naeem et al., 1994; Gross and Cardinale, 2007; Hillebrand and Matthiessen, 2009). It is easy to understand that all these avenues explain essential, but not adequate, range to demonstrate the abundance-diversity relationships in disposition. Cardinale et al. (2009) proposed that recognized diversity richness-abundance relationships are built by the interaction of four specific, non-compatible variables: quantity of confine resources, resource inequality, and species richness, and tested this access with practical, substantial phytoplankton data (Lehtinen et al., 2017). They highlighted that experimentally controlled research, with controls of resource proportion, their ratios, and species richness would be probable to check the combined influence of the factors on biomass and abundance. The present study revealed that abundance had a significant effect on the species richness and diversity has been shown also to hike the resistance of plankton communities (Ptacnik et al., 2008). The results of this study alone has narrow descriptive strength for forecasting of winner species in planktonic windows of possibility, and for the outcomes of events of resource domination, in the paucity of quantitative data on their ecological characteristics. These features comprise explanation of competitive might within a set of environmental context, but also the "consumer standpoint (Litchman and Klausmeier 2008; Vallina et al., 2014). Inconsistent to many earlier investigations on biodiversity-ecosystem active interrelation, we found a highly symbolic inverse relationship between abundance and species richness. This indicates that species-rich natural phytoplankton communities form the basis for opportunistic species to temporarily monopolize resources and create blooms.

Kuwait's presumably unspoilt environment is intimidated by possible naturally associated influences that development may have on this ecological region. Among these hazards are loss of vital habitat to development, contamination due to poisonous chemicals associated with oil exploration, invasive species from ballast waters and eutrophication (Ibrahim and El Samra, 1987; Al-Muzaini and Jacob, 1996; Subba-Rao et al., 1999; ROPME, 1999; Nour El-Din and Al-Khayat, 2005; Sheppard et al., 2010). Moreover, "brine" released from desalination plants with salinities $>75$ psu has increase salinity in already hypersaline water bodies, especially during summer due to high evaporation leading to higher salinity as seen during events 9-12 in this study (Abdul Azis et al., 2003; Lattemann and Hopner, 2008; Sheppard et al., 2010). The temporal variations in abundance and species richness of the phytoplankton community show that physico-chemical factors and seasons play a major role in the functioning of this evidently emphatic ecosystem.

Further detailed studies are needed in other subtidal waters of North-western Arabian Gulf, mainly in the north and south of Kuwait, considering phytoplankton ecology and species composition including benthic diatoms and the effect of pollution. Also, increasing the study sites in future; extending to different areas north and south is needed in order to compare the diversity and species composition in the study area. Divergence in the phytoplankton groups reflects the seasonal fluctuations and impact of water quality. Presence of potentially harmful algal species even in low counts, make the region susceptible to harsh effects due to flourishing of these species during favorable conditions. Continuous monitoring of this area is essential to follow probable bloom of these species, to predict negative effects deriving from developing land activities, anthropogenic inputs and climate change.

\section{Acknowledgments}

The authors express their sincere gratitude to the field work team for assistance in the field. This work was a part of the research project entitled: "Ecological assessment and restoration plan of terrestrial biodiversity in Umm Al-Namil Island, Kuwait". The project was funded by the Kuwait Foundation for the Advancement of Sciences (KFAS) under project code: FA121C.

\section{References}

Abdul Azis, P.K., I.A.Al-Tisan, M.A. Daili, T.N. Green, A.G.I. Dalvi and M.A. Javeed: Chlorophyll and plankton of the Gulf coastal waters of Saudi Arabia bordering a desalination plant. Desalination, 154, 291-302 (2003).

Abdulaziz, P., I. Al-Tisan, M. Al-Daili, T. Green, A. Ghaniand and M. Javeed: Effects of environment on source water for desalination plants on the eastern coast of Saudi Arabia. Desalination, 132, 29$40(2000)$.

Ahmed, A. and A. Wanganui: Phytoplankton succession in a tropical freshwater lake, Bhoj Wetland (Bhopal, India): Spatial and temporal perspective. Environ. Monit. Assess., 187, 192 (2015).

Al-Muzaini, S. and P. G. Jacob: Marine plants of the Arabian Gulf. Environ. Int., 22, 369-376 (1996).

Ali, M. Arabian: Gulf food web and the effect of salinity on feeding and growth rate of young three spine sticklebacks (Gasterosteus aculeatus). University of Sheffield, Sheffield, England, UK (2015).

Ali, M., M. Al-Mutairi, M.N.V. Subrahmanyam, S. Isath, M. A. AlAwadi, P.N. Kumar, K. Al-Hebini and S. A. S. Omar: Temporal variations in abundance and species richness of zooplankton with emphasis on ichthyoplankton in the subtidal waters of Umm Al-Namil Island, Northwestern Arabian Gulf of the ROPME Sea Area. Am. J. Environ. Sci., 15, 188-204 (2020).

Al-Yamani, F.Y., D.V. Subba Rao, W. Ismail, K. Al-Rifaie, H. Al-Mutairi, A.Al-Ghunaim, A. Lennox, C.V. Nageswara Rao, M.N.V. Subrahmanyam and J.M. Al- Hassan: Did algal blooms cause fish kills off Kuwait? International Conference on Harmful Algal Blooms, Tasmania, p. 309 (2000).

Al-Yamani, F., M. Saburova and I. Polikarpov: Apreliminary assessment of harmful algal blooms in Kuwait's marine environment. Aquat. Ecosyst. Hlth., 15, 64-72 (2012).

Al-Yamani, F.Y., T. Yamamoto, T. Al-Said and A. Al-Ghunaim: Dynamic hydrographic variations in northwestern Arabian Gulf over the past three decades: Temporal shifts and trends derived from long-term monitoring data. Mar. Pollut. Bull., 122, 488-499 (2017).

Al-Zamel, A., M. Al-Sarawi and S. Khader: Coastal geomorphology, hydrodynamics and biolithofacies in the intertidal and subtidal areas of Umm Al-Namil Island, Kuwait Bay, Kuwait. J. Coastal Res., 23, 501-514 (2007).

Badsi, H., H.O. Ali, M. Loudiki and A. Aamir: Phytoplankton diversity and 
community composition along the salinity gradient of the mass estuary. Am. J. Hum. Ecol., 1, 58-64 (2012).

Boyd, P.W., T. A. Rynearson, E. A. Armstrong, F. Fu, K. Hayashi, Z. Hu, D. A. Hutchins, R.M. Kudela, E. Litchman, M.R. Mulholland, U. Passow, R.F. Strzepek, K.A. Whittaker, E. Yu and M.K. Thomas: Marine phytoplankton temperature versus growth responses from polar to tropical waters-outcome of a scientific community-wide study. PLOS One, 8, e63091 (2013).

Butterwick, C., S.I. Heaney and J.F. Talling: Diversity in the influence of temperature on the growth rates of freshwater algae, and its ecological relevance. Freshwat. Biol., 50, 291-300 (2005).

Buzzi, F.: Phytoplankton assemblages in two sub-basins of lake Commo. J. Limnol., 61, 117-128 (2002).

Calliari, D., M. Gomez and N. Gomez: Biomass and composition of the phytoplankton in the Río de la Plata: Large-scale distribution and relationship with environmental variables during a spring cruise. Cont. ShelfRes., 25, 197 (2005).

Canale, R.P. and A.H. Vogel: Effects of temperature on phytoplankton growth. J. Environ. Eng., 100, 231-241 (1974).

Cardinale, B. J., D. M. Bennett, C. E. Nelson and K. Gross: Does species diversity drive productivity or vice versa? A test of the multivariate productivity-diversity hypothesis in streams. Ecology, 90,12271241 (2009).

Cardoso, S.J., F. Roland, S.M.L. Oliveira and V.L.M. Huszar: Phytoplankton abundance, biomass and diversity within and between Pantanal wetland habitats. Limnology, 42, 235-41 (2012).

Castillo, FA and Z. Vizcaino: Observation of the Pacific phytoplankton during 1991-1992 in El Niño conditions. Bull. Inst. Fr. Études Andines, 22, 179-190 (1993).

Chakraborty, A., G. Padmavati and A.K. Gosh: Tidal variation of phytoplankton in the coastal waters of South Andaman, India. J. Environm. Biol., 36, 207-214 (2015).

Chapin, F.S., E.S. Zavaleta, V.T. Eviner, R.L. Naylor, P.M. Vitousek, H.L. Reynolds, D.U. Hooper, S. Lavorel, O.E. Sala, S.E. Hobbie, M.C. Mack and S. Diaz: Consequences of changing biodiversity. Nature., 405, 234-242 (2000).

Chen, Y.L.: Spatial and seasonal variations of nitrate-based new production and primary production in the South China Sea. Deep Sea Res., 1, 319-340 (2005).

De Domitrovic, Y.Z., A.S.G.P. de Neiff and S.L. Casco: Abundance and diversity of phytoplankton in the Paraná River (Argentina) $220 \mathrm{~km}$ downstream of the Yacyretá reservoir. Braz. J. Biol., 67, 53-63 (2007).

Devlin, M.J., M. Breckels, C.A. Graves, J. Barry, E. Capuzzo, F.P. Huerta, F. Al-Ajmi, M.M. Al-Hussain, W.J.F. LeQuesne and B.P. Lyons: Seasonal and temporal drivers influencing phytoplankton community in Kuwait marine waters: Documenting a changing landscape in the Gulf. Front. Mar. Sci., 6, 1-22 (2019).

El-Bassat, R.A. and W.D. Taylor: The zooplankton community of Lake Abo Zaabal, a newly-formed mining lake in Cairo, Egypt. Afr. J. Aquatic Sci., 32, 1-8 (2007).

El Gammal, M.A.M., M. Nageeb and S. Al-Sabeb: Phytoplankton abundance in relation to the quality of the coastal water-Arabian Gulf, Saudi Arabia. Egyptian J. Aquatic Res., 43, 275-282 (2017).

Gross, K. and B.J. Cardinale: Does species richness drive community production or vice versa? Reconciling historical and contemporary paradigms in competitive communities. Am. Nat., 170, 207-220 (2007)

Hillebrand, $\mathrm{H}$. and B. Matthiessen: Biodiversity in a complex world: Consolidation and progress in functional biodiversity research. Ecol. Lett., 12, 1405-1419 (2009).
Hinga, K.R.: Effects of pH on coastal marine phytoplankton. Mar. Ecol. Prog. Ser., 238, 281-300 (2002).

Hoekstra, A. Y., M. M. Mekonnen, A. K. Chapagain, R. E. Mathews and B. D. Richter: Global monthly water scarcity: Blue water footprints versus blue water availability. PLoS ONE, 7, e32688 (2012).

Hooper, D. U.,F. S. Chapin, J. J. Ewel, A. Hector, P. Inchausti, S. Lavorel, J. H. Lawton, D. M. Lodge, M. Loreau, S. Naeem, B. Schmid, H. Setälä, A. J. Symstad, J. Vandermeer and D. A. Wardle: Effects of biiodiversityon ecosystem functioning: A consensus of current knowledge. Ecol. Monog., 75, 3-35 (2005).

IPCC.: Climate Change: Synthesis Report. IPCC, Geneva (2007).

Ibrahim, M. A. and M.I. El Samra: Inhibition effect of four oil dispersants on primary productivity in Qatar water (ArabianGulf). Qatar Univ. Sci. Bull., 7, 379-391(1987).

Khan, T.A.: Limnology of four saline lakes in western Victoria, Australia. Limnologica, 33, 327-339 (2003).

Khomayis, H.S.: The annual of nutrient salts and chlorophyll a in the coastal waters of Jeddah, Red Sea. JKAU: Mar. Sci., 13, 131-145 (2002).

Langlois, G.W. and P. Smith: Phytoplankton In: Biology and Ecological Niches in the Gulf of the Farallones: U.S. Geological Survey, Reston, Virginia, pp. 32-35 (2001).

Lattemann, S. and T. Hopner: Environmental impact and impact assessment of seawater desalination. Desalination, 220, 1-15 (2008).

Lehtinen, S., T. Tamminen, R. Ptacnik and T. Andersen: Phytoplankton species richness, evenness, and production in relation to nutrient availability and imbalance. Limnol. Oceanogr., 62, 1393-1408 (2017).

Litchman, E. and C.A. Klausmeier: Trait-based community ecology of phytoplankton. Annu. Rev. Ecol. Evol. Syst., 39, 615-639 (2008).

Lovejoy, T.E. and L.J. Hannah: Climate change and biodiversity. Yale University Press, Yale, pp. 440 (2006).

McNaughton, S.J.: Diversity and stability of ecological communitiescomment on role of empiricism in ecology. Am. Nat., 111, 515-525 (1977).

MOOPAM (Manual of Oceanographic Observations and Pollutant Analyses Methods):: Fourth Edition, ROPME (Regional Organization for the Protection of the Marine Environment), Kuwait (2010).

Morais, P., M.A. Chícharo and A. Barbosa: Phytoplankton dynamics in a coastal saline lake (SE-Portugal). Acta Oecol., 24, 87-96 (2003).

Moreno-Díaz, G., A. A. Rojas-Herrera, J.Violante-González, J. González-González, J. L. R. Acevedo and S. G. Ibáñez: Temporal variation in composition and abundance of phytoplankton species during 2011 and 2012 in Acapulco Bay, Mexico. Open J. Mar. Sci., 5,358-367 (2015).

Naeem, S., L.J. Thompson, S.P. Lawler, J.H. Lawton and R.F. Woodfin: Declining biodiversity can alter the performance of ecosystems. Nature, 368, 734-737 (1994).

Naeem, S. and S. Li: Biodiversity enhances ecosystem reliability. Nature, 390, 507-509 (1997).

Nassar, M.Z.A. and S.M. Gharib: Spatial and temporal patterns of phytoplankton composition in Burullus Lagoon, Southern Mediterranean Coast, Egypt. J. Aquat. Res., 40, 133-42 (2014).

Newall, E.J.L., V.T. Chu, O. Pringault, D. Amouroux, R. Arfi, Y. Bettarel, T. Bouvier, C. Bouvier, P. Got, T.M.H. Nguyen, X. Mari, P. Navarro, T.N. Duong, T.T.T. Cao, T.T. Pham, S. Ouillon and J.P. Torreton: Phytoplankton diversity and productivity in a highly turbid, tropical coastal system (Bach Dang Estuary, Vietnam). Biogeosci. Discuss., 8, 487-525 (2001).

Nour El-Din, N.M. and J.A. Al-Khayat: Phytoplankton-zooplankton 
relations in three inland seas along the Qatari Coast (ArabianGulf). Int. J. Environ. Stud., 62, 375-390 (2005).

Örnólfsdóttir, E.B.,E. Lumsden and J.L. Pinckney: Phytoplankton community growth-rate response to nutrient pulses in a shallow turbid estuary, Galveston Bay, Texas. J. Plankton Res., 26, 325-339 (2004).

Parnell, P.E.: The effects of sewage discharge on water quality and phytoplankton of Hawaiian coastal waters. Mar. Environ. Res., 55, 293-311 (2003).

Polikarpov, I., F. Al-Yamani and M. Saburova: Space-time variability of phytoplankton structure and diversity in the north-western part of the Arabian Gulf (Kuwait's waters). In: Proceedings of the First Middle Eastern Biodiversity Congress Environment, Biodiversity and Conservation in the Middle East, Aqaba, pp. 20-23 (2008).

Price, A.R.G.: Temporal variations in abundance of penaeid shrimp larvae and oceanographic conditions of Ras Tanura. Western RSA. Estuar. Coast. Mar. Sci., 9, 451-465 (1979).

Ptacnik, R., A. G. Solimini, T. Andersen, T. Tamminen, P. Brettum, L. Lepisto, E. Willen and S. Rekolainen: Diversity predicts stability and resource use efficiency in natural phytoplankton communities. Proc. Natl. Acad. Sci., USA 105, 5134-5138 (2008).

QMEMP(Qatar Marine Environment Monitoring Program): Prepared by Technical Affairs Department, Supreme Council for the Environment and Natural Reserves (Currently Ministry of Environment), State of Qatar, pp. 54 (2005).

Quigg, A., M. Al-Ansi, N. Nour Al Din, C.L. Wei, C.C. Nunnally, I.S.AlAnsari, G.T. Rowe, Y. Soliman, I. Al-Maslamani, I.Mahmoud, N. Youssef and M.A. Abdel-Moati: Phytoplankton along the coastal shelf of an oligotrophic hypersaline environment in a semienclosed marginal sea: Qatar (Arabian Gulf). Cont. Shelf Res., 60, 1-16 (2013).

$R$ CORE TEAM: R: A language and environment for statistical computing. R Foundation for Statistical Computing, Vienna, Austria (2016). https://www.R-project.org/

Rojas-Herrera,A. A., J. Violante-González, V. M. G. Sevilla-Torres, J. S. G. Guerrero, P. Flores-Rodríguez and J.A.O. Rendón-Dircio: Species composition and abundance of phytoplankton communities in Acapulco Bay, Mexico. Int. Res. J. Microbiol., 3, 307-316 (2012a).

Rojas-Herrera, A. A., J. Violante-González, S. García-lbáñez, V. M. G. Sevilla-Torres, J. S. Gil-Guerrero and P. Flores-Rodríguez: Temporal variation in the phytoplankton community of Acapulco Bay, Mexico. Microbiol. Res.,3, e4 (2012b).

ROPME (Regional Organization for the Protection of the Marine Environment):: Regional Report of the State of the Marine Environment, Regional Organization for the Protection of the Marine Environment, State of Kuwait (1999).

Scheffer, M.: Should we expect strange attractors behind plankton dynamics-and if so, should we bother? J. Plankton Res., 13, 1291-1305 (1991).

Schumann, R.,H. Baudler, A. Glass, K. Dümcke and U. Karsten: Long-term observations on salinity dynamics in a tideless shallow lagoon of the Southern Baltic Sea coast. J. Mar. Syst., 60, 330-344 (2006).

Sheppard, C., M. Al-Husiani, F. Al-Jamali, F. Al-Yamani, R. Baldwin, J. Bishop, F. Benzoni, E. Dutrieux, N.K. Dulvy, D.V. Subba Rao, D.A. Jones, R. Loughl and D. Medio, M. Nithyanandan, G. M. Pilling, I. Polikarpov, A.R. Price, S. Purkis, B. Riegl, M. Saburova, K.S. Namin, O. Taylor, S. Wilson and K. Zainal: The Gulf: Ayoung sea in decline. Mar. Pollut. Bull., 60, 13-38 (2010).

Subba-Rao, D.V., F. Al-Yamani, A. Lennox, Y. Pan and T.F.O. As-Said: Biomass and production characteristics of the first red tide notice din KuwaitBay, Arabian Gulf. J. Plankton Res., 21, 805-810 (1999).

Tomas,C.R.: Identifying Marine Phytoplankton, Academic Press (1997).

Touliabah, H.E., R.A. Elbassat, A.J. Turki, M.A. Affan, M.S. Hariri, R.M.E. Hassanine, N.I.H. Abdulwassi and A.W. Almutairi: Plankton and some environmental variables as a water quality indicator for saline pools at the Eastern Red Sea (Saudi Arabia). J. Coast Zone Manag., 19, 417 (2016).

Touliabah, H.E., W.S. Abu EL-Kheir, K.M.G. Kuchari and N.H. Abdulwassi: Phytoplankton composition at Jeddah Coast-Red Sea Saudi Arabia in relation to some ecological factors. JKAU: Sci., 22, 115-131 (2010).

Turkoglu, M.: Temporal variations of surface phytoplankton, nutrients and chlorophyll a in the Dardanelles (Turkish Straits System): A coastal station sample in weekly time intervals. Turk. J. Biol., 34, 319-333 (2010).

Utermöhl, H.: Zur Vervollkommnung der quantitativen phytoplanktonmethodik. Mitt. Int.Ver. Limnol., 9, 1-38 (1958).

Vallina, S.M., M.J. Follows, S. Dutkiewicz, J.M. Montoya, P. Cermeno and M. Loreau: Global relationship between phytoplankton diversity and productivity in the ocean. Nat. Commun., 5, 4299 (2014).

Varela, M. and R. Prego: Hydrography and phytoplankton in an isolated and non pristineria area: The Coruña harbour (NW Spain). Acta Oecologica, 24, 113-124 (2003).

Varona-Cordero, F., F. Gutierrez-Mendieta and V.H. Rivera-Monroy: Insitu response of phytoplankton to nutrient Additions in a tropical coastal lagoon, (La Mancha, Veracruz, Mexico). Estuar. Coast., 37, 1353-1375 (2014).

Webb, B.W. and F. Nobilis: Long-term changes in river temperature and the influence of climatic and hydrological factors. Hydrolog. Sci. J., 52, 74-85 (2007).

Winder, M. andJ.E.Cloern: The annual cycles of phytoplankton biomass. Philos. Trans. R. Soc. Lond., 365, 3215-3226 (2010).

Worm, B., E.B. Barbier, N. Beaumont and J.E. Duffy: Impacts of biodiversity loss on ocean ecosystem services. Science, 314, 787-790 (2006). 